Pacific Journal of Mathematics 


\title{
BORSUK-ULAM THEOREM, FIXED POINT INDEX AND CHAIN APPROXIMATIONS FOR MAPS WITH MULTIPLICITY
}

\author{
Fritz von HAESEler AND GeNCHO SKordeV
}

\begin{abstract}
In this article we consider $m$-acyclic maps with respect to a field $\mathbb{F}$ and prove the existence of chain approximation for such maps. Furthermore we provide a unified approach to the Borsuk-Ulam theorem and the Bourgin-Yang generalization. Finally we prove the existence of $A$-systems for certain $m$-acyclic maps and define a fixed point index.
\end{abstract}

There are essentially two ways to handle multivalued fixed point or coincidence problems. The first one is based on homological arguments (homological method) and the second on the homotopical method where the multivalued map is approximated with single valued maps. For a survey of both methods we recommend [3], for single valued maps we refer to $[4,8]$.

The homological method also splits in two directions-the considerations on the level of homology groups and chain approximation techniques. For the first one see [3], the second one-chain approximations of multivalued maps-has roots in the early work of $\mathrm{L}$. Vietoris (see [1] where the Vietoris-Begle mapping theorem is proved). The chain approximation technique is used by S. Eilenberg and D. Montgomery [10] to prove a Lefschetz fixed point theorem for acyclic maps on compact ANR's. B. O'Neil constructed chain approximations for a more general mapping class, i.e. $(1, n)$-mappings, and also proved a Lefschetz fixed point theorem for such mappings on polyhedra [22]. The same technique was the main tool for developing the fixed point index with all properties (including commutativity and mod-p-property ; multiplicity is proved in [26]) for multivalued maps of ANR's ([9, 11, 25]). The main result in [25] may be stated as follows: If a class of multivalued maps has arbitrarily close chain approximations, then there is a fixed point index with all properties for this class.

In this paper we consider so called $m$-acyclic maps with respect to a given field $\mathbb{F}$ and prove that for such mappings there exist chain 
approximations. $m$-maps are also called acyclic carriers and are investigated in [5, 6]. Therefore from [25], for $m$-acyclic maps there is also a fixed point index with all properties. The class of $m$-acyclic maps includes $\mathbb{F}$-acyclic maps, $(1, n)$-maps [22], single valued maps with values in symmetric products of a space [20], $m$-maps [16], weighted maps [7], and others.

We also provide a unified approach to Borsuk-Ulam theorems for single valued mappings as well as for $m$-acyclic maps with respect to $\mathbb{Z}_{2}$ via chain approximations. The Borsuk-Ulam theorem or antipodal theorem can be stated in several ways; we prefer the following formulation: If $f: S^{n} \rightarrow \mathbb{R}^{n}$ is a continuous map, then there exists a point $x \in S^{n}$ such that $f(x)=f(-x)$, see [30]. We prove that this theorem holds for every multivalued map $F: S^{n} \rightarrow \mathbb{R}^{n}$ which has arbitrarily close chain approximations with odd Kronecker index. Therefore the Borsuk-Ulam theorem is true for $m$-acyclic maps w.r.t. $\mathbb{Z}_{2}$, especially for $(1,2 k+1)$-maps. This is a partial answer to a question of $\mathrm{H}$. Schirmer in [23]; see also [15] for acyclic maps.

There are various generalizations and applications of Borsuk-Ulam theorems (cf. [30] for a good review). We shall consider also the Bourgin-Yang generalization of the Borusk-Ulam theorem, see [12, 30]. If $f: S^{n+k} \rightarrow \mathbb{R}^{n}, k \geq 0$, is a continuous map and $B(f)=$ $\left\{x \in S^{n+k} \mid f(x)=f(-x)\right\}$ then $\operatorname{dim} B(f) \geq k$, or, more generally $g(B(f)) \geq k$, where $g(B(f))$ is the genus of the space $B(f)$ with the antipodal involution $x \mapsto-x$, cf. [17, 30]. The Bourgin-Yang theorem is proved for acyclic (or admissible maps) in [12].

We shall show that the Bourgin-Yang theorem holds for all multivalued maps $F: S^{n+k} \rightarrow \mathbb{R}^{n}, k \geq 0$, having arbitrarily closed chain approximations with odd Kronecker index. Therefore the BourginYang theorem is true for $m$-acyclic maps w.r.t. $\mathbb{Z}_{2}$.

Acknowledgment. We would like to thank Professor H.-O. Peitgen for valuable discussions and for helpful suggestions.

\section{Preliminaries.}

1. Multivalued maps with multiplicity. We consider multivalued maps $F: K \rightarrow L$ [2] for which $F(x)$ is a nonempty compact subset of $L$ for each $x \in K$. Two points $\left(x_{i}, y_{i}\right) \in \Gamma(F)=\{(x, y) \mid x \in K$, $y \in F(x)\} \subset K \times L, i=1,2$, are equivalent $\left(\left(x_{1}, y_{1}\right) \sim\left(x_{2}, y_{2}\right)\right)$ if and only if $x_{1}=x_{2}$ and $y_{1}, y_{2}$ are in the same connected component of $F(x)$. This defines a new set $\widetilde{\Gamma}(F)=\Gamma(F) / \sim$, with elements denoted by $(x, C(x)) ; C(x)$ denotes also a connected component of $F(x)$ as a subset of $L$. 
By $\mathbb{A}$ we denote a ring without zero divisors, and by $\mathbb{F}$ we denote a field.

Definition 1. Let $F: K \rightarrow L$ be a multivalued map and $\mathbb{A}$ a ring without zero divisors. A map $m: \widetilde{\Gamma}(F) \rightarrow \mathbb{A}$ is called a multiplicity function.

Definition $2([5,6])$. Let $F: K \rightarrow L$ be a multivalued map with multiplicity-function $m: \widetilde{\Gamma}(F) \rightarrow \mathbb{A} . F$ is an $m-$ map (w.r.t. $\mathbb{A}$ ) if the following two conditions are satisfied:

1. $F(x)$ consists of finitely many connected components for each $x \in K$.

2. For all $x_{0} \in K$ with $F\left(x_{0}\right)=C_{1}\left(x_{0}\right) \cup \cdots \cup C_{s}\left(z_{0}\right), s=s\left(x_{0}\right)$, and disjoint neighbourhoods $U_{i}$ of $C_{i}\left(x_{0}\right)$ there exists a neighbourhood $U$ of $x$ such that (a) $F(U) \subset \bigcup_{i=1}^{s} U_{i}$, and (b) $m\left(x_{0}, C_{i}\left(x_{0}\right)\right)=$ $\sum_{C(x) \subset U_{i}} m(x, C(x))$ for all $x \in U$ and $i=1, \ldots, s$.

REMARK 1. If $K$ is connected and $x_{1}, x_{2} \in K$ then

$$
\sum_{C\left(x_{1}\right)} m\left(x_{1}, C\left(x_{1}\right)\right)=\sum_{C\left(x_{2}\right)} m\left(x_{2}, C\left(x_{2}\right)\right),
$$

where the summation is taken over the connected components of $F\left(x_{i}\right), i=1,2$ (cf. Lemma 2.3 in [16]). Therefore, for $K$ connected it makes sense to speak of the multiplicity of the $m$-map $F$, $m(F)=\sum_{c(x)} m(x, C(x))$, which is well defined.

In all that follows we suppose $K$ to be connected.

Definition 3. Let $F: K \rightarrow L$ be an $m$-map with multiplicity $m(F)=a \neq 0$. Then $F$ is called an $m$-acyclic map (w.r.t. $\mathbb{A}$ ) if for each $x \in K$ the connected components $C(x)$ of $F(x)$ are acyclic compact sets with respect to Čech homology with coefficients in $\mathbb{A}$, i.e., $\widetilde{H}_{i}(C(x), \mathbb{A})=0, i \geq 0$.

If for each $x \in K$ the connected components of $F(x)$ are points we call $F$ an $m$-point map (w.r.t. $\mathbb{A}$ ).

REMARK 2. If $F$ is an $m$-acyclic map with multiplicity $m(F) \neq 0$ w.r.t. the field $\mathbb{F}$, then we may consider $F$ as an $m$-acyclic map with multiplicity 1 and multiplicity-function

$$
m_{1}(x, C(x))=m(x, C(x)) m(F)^{-1} .
$$

We assume that $m$-acyclic maps (w.r.t. a field $\mathbb{F}$ ) have multiplicity 1 . 
EXAMPLES. 1. Acyclic [10] or single valued maps are $m$-acyclic maps, $m$-point maps resp. with multiplicity-function $m(x, C(x)) \equiv$ 1 .

2. $(1, n)$-maps $F[9,22]$, with $n \equiv 1(\bmod 2)$ are $m$-acyclic maps w.r.t. $\mathbb{Z}_{2}$ and multiplicity-function $m(x, y) \equiv 1$ for all $x, y \in \widetilde{\Gamma}(F)$.

3. Let $R: \widehat{\mathbb{C}} \rightarrow \widehat{\mathbb{C}}, \widehat{\mathbb{C}}=\mathbb{C} \cup\{\infty\}$, be a rational map; then the map $R^{-1}(z)=\{w \in \widehat{\mathbb{C}} \mid R(w)=z\}$ is an $m$-point map w.r.t. $\mathbb{Z}$ and multiplicity-function $m(z, w)=$ local degree of $R$ at $w$. Furthermore, $m\left(R^{-1}\right)=d$, the degree of $R$. For polynomials cf. [14].

4. Let $\widetilde{X}, X$ be finite polyhedra and $\pi: \widetilde{X} \rightarrow X$ a ramified covering with multiplicity map $\tilde{\mu}: \widetilde{X} \rightarrow \mathbb{N}$, see [28]. The map $\pi^{-1}: X \rightarrow \widetilde{X}$ is an $m$-point map (w.r.t. $\mathbb{Z}$ ) with multiplicity-function $\mu: \widetilde{\Gamma}\left(\pi^{-1}\right) \rightarrow \mathbb{Z}$, $\mu(x, \tilde{x})=\tilde{\mu}(\tilde{x})$.

5. Let $X$ be a $G$-space and $|G|<\infty, G_{x}$ denotes the stability subgroup of $x, G_{x}=\{g \in G \mid g x=x\}$. Then the inverse map, $\pi^{-1}$, of the projection $\pi: X \rightarrow X / G$ is an $m$-point map w.r.t. $\mathbb{Z}$. If $\pi^{-1}(y)=\left\{x_{1}, \ldots, x_{s}\right\}, s=s(y)$ the multiplicity-function is defined by

$$
m\left(y, x_{i}\right)=\left|G_{x_{i}}\right| \text {. }
$$

6. Let $f: K \rightarrow S P^{n} L, n \in \mathbb{N}$, be a single valued map; $S P^{n} L$ denotes the $n$-th symmetric product of $L$ [20]. Then $f$ induces an $m$-point map $F$ w.r.t. $\mathbb{Z}$ which is defined by

$$
F(x)=\pi(f(x)),
$$

with $\pi: S P^{n} L \rightarrow L, \pi\left(x_{1}^{k_{1}} \cdots x_{s}^{k_{s}}\right)=\left\{x_{1}, \ldots, x_{s}\right\}, \sum k_{i}=n$. The multiplicity-function is given by $m\left(x, x_{i}\right)=k_{i}$ and $F$ has multiplicity $n$.

On the other hand, if $F: K \rightarrow L$ is an $m$-point map w.r.t. $\mathbb{Z}$ with multiplicity $m(F)=n>0$ and $m(x, C(x)) \geq 0$ for all $x \in K$, then this induces a single valued map $\widehat{F}: K \rightarrow S P^{n} L$ defined by $\widehat{F}(x)=$ $x_{1}^{k_{1}} \ldots x_{s}^{k_{s}}, s=s(x)$, and $F(x)=\left\{x_{1}, \ldots, x_{s}\right\}, k_{i}=m\left(x, x_{i}\right)$.

More examples may be found in [5], [21], [28].

2. Block complexes. We shall use a special kind of CW-complexes, namely block-complexes, cf. $[19$, p. 134] (it is possible to work with regular CW-complexes $[29$, p. 60$])$.

Definition 4. Let $K$ be a finite simplicial complex with fixed triangulation $\bar{\tau}$. An $n$-block (w.r.t. $\mathbb{A}$ ) in $K$ is a pair of subcomplexes $(e, \dot{e})$ in $\bar{\tau}$ such that $\dot{e} \subset e, \operatorname{dim} e=n, \operatorname{dim} \dot{e}=n-1$ and $H_{s}(e, \dot{e})=$ $H_{s}\left(B^{n}, \dot{B}^{n}\right)$ for every $s \geq 0\left(B^{n}\right.$ denotes the $n$-dimensional ball and 
$\dot{B}^{n}$ its boundary, homology with coefficients in $\mathbb{A}$ ). The subcomplex $\dot{e}$ is called boundary of the block $e$, and $\operatorname{Int}(e)=e \backslash \dot{e}$ is called interior of $e$.

Definition 5. A block dissection (b.d.) $\tau$ of the triangulation $\bar{\tau}$ is a set of blocks (w.r.t. $\mathbb{A}$ ) of $\bar{\tau}$ such that

(a) every simplex of the triangulation $\bar{\tau}$ is in the interior of just one block of $\tau$, and

(b) the boundary $\dot{e}$ of each block $e$ of $\tau$ is a union of $(\operatorname{dim} e-1)$ blocks.

If in the simplicial complex $K$ with given triangulation $\bar{\tau}$ a b.d. $\tau$ is fixed, we call $K$ a block complex with block structure $\tau$, and write $(K, \tau)$.

RemarK 3. The block complex $K$ is a regular CW-complex, [29, p. 60]. The mesh of the block complex $(K, \tau)$ is defined as $\operatorname{mesh}(K, \tau)$ $=\max \{\operatorname{diam}(e) \mid e \in \tau\}$ (we consider the metric in $K$ defined by barycentric coordinates of the triangulation $\bar{\tau}$ of $K$ ).

We shall consider block complexes w.r.t. a ring $\mathbb{A}$ or a field $\mathbb{F}$.

Definition 6. Let $(K, \tau)$ be a block complex of the simplicial complex with triangulation $\bar{\tau}$ and let $\tau_{1}$ be a block structure of a subdivision $\bar{\tau}_{1}$ of the triangulation $\bar{\tau}$. The block structure $\tau_{1}$ is called a subdivision of the block structure $\tau$ if every block of $\tau_{1}$ is in the interior of just one block of $\tau$. In this case we write $\tau_{1}>\tau$.

Definition 7. Let $\tau_{1}$ be a b.d. of the triangulation $\bar{\tau}_{1}$. The sequence $\hat{\tau}=\left\{\tau_{i}\right\}$ of block subdivisions of $\tau_{1}$ is called a fundamental sequence of block dissections (f.s.b.d.) with respect to $\tau_{1}$ if:

(a) $\tau_{i+1}$ is a subdivision of $\tau_{i}, i \geq 0$, and

(b) $\lim _{i \rightarrow \infty} \operatorname{mesh}\left(K, \tau_{i}\right)=0$.

Let $\tau$ be a b.d. (w.r.t. $\mathbb{A}$ ) of the triangulation $\bar{\tau}$. We denote by $C_{*}(K, \tau)$ or $C_{*}(\tau)$ the chain complex of the block complex $\tau$ with coefficients in a given ring $\mathbb{A}$ (the chain complex of the block-complexes $(K, \tau),[19])$.

For $M \subset K$ we define $S t(M, \tau)=\bigcup\{e \in \tau \mid e \cap M \neq \varnothing\}$ and $S t^{k+1}(M, \tau)=S t\left(S t^{k}(M, \tau), \tau\right)$ for $k \in \mathbb{N}$.

For a given b.d. $\tau_{1}$ and its block subdivision $\tau_{2}$ there is a chain map (chain subdivision)

$$
b\left(\tau_{1}, \tau_{2}\right): C_{*}\left(\tau_{1}\right) \rightarrow C_{*}\left(\tau_{2}\right)
$$

such that (a) $b\left(\tau_{1}, \tau_{2}\right)(e)=e$ for every 0 -block, and (b) $\left|b\left(\tau_{1}, \tau_{2}\right)(e)\right|$ $\subset e$ for every block $e \in \tau,[26]$. Here $|c|$ denotes the support of the 
chain $c \in C_{*}(\tau)$, i.e., the smallest block subcomplex $K^{\prime} \subset K$ of the block structure $\tau$ such that $c \in C_{*}\left(K^{\prime}, \tau\right)$.

There exists a chain map (chain approximation of the identity)

$$
\chi\left(\tau_{2}, \tau_{1}\right): C_{*}\left(\tau_{2}\right) \rightarrow C_{*}\left(\tau_{1}\right)
$$

such that (a) $\chi\left(\tau_{2}, \tau_{1}\right)(e)$ is a 0-block in $\tau_{1}$ for every 0-block $e \in \tau_{2}$, and (b) $\left|\chi\left(\tau_{2}, \tau_{1}\right)(e)\right| \subset|e|$ for all blocks $e \in \tau_{2}$, where $|e|$ is the support of the block $e$ in $\tau_{1}$, i.e., $|e|$ is the unique block in $\tau_{1}$ such that $e \subset \operatorname{Int}|e|$.

The chain map $\chi\left(\tau_{2}, \tau_{1}\right)$ is not unique but every two such maps are chain homotopic with a chain homotopy $D$ such that $|D(e)| \subset|e|$, [26].

If $\hat{\tau}=\left\{\tau_{i}\right\}$ is a f.s.b.d. of $K$ we shall consider

$$
C_{*}(\hat{\tau})=\left\{C_{*}\left(\tau_{i}\right), b\left(\tau_{i}, \tau_{i+1}\right), \chi\left(\tau_{i+1}, \tau_{i}\right), i=0,1, \ldots\right\}
$$

with fixed chain maps $\chi\left(\tau_{i+1}, \tau_{i}\right)$. If the f.s.b.d. is clear from the context we use the notation $b(i, i+1)$ and $\chi(i+1, i)$, and for $k<$ $l$ we denote the chain subdivision map from $C_{*}\left(\tau_{k}\right)$ in $C_{*}\left(\tau_{l}\right)$ by $b(k, l)=b(l-1, l) b(l-2, l-1) \cdots b(k, k+1)$ and by $\chi\left(\tau_{l}, \tau_{k}\right)=$ $\chi(k-1, k) \cdots \chi(l, l-1)$ the chain map from $C_{*}\left(\tau_{l}\right)$ in $C_{*}\left(\tau_{k}\right)$.

3. Chain approximation of upper semicontinuous maps.

Definition 8. Let $K$ and $L$ be finite simplicial complexes with given block structures $\tau_{1}, \tau_{2}, \tau_{2}>\tau_{1}$ on $K$, and $\mu$ on $L$ (w.r.t. $\mathbb{A}$ ). Let $F: K \rightarrow L$ be an upper semicontinuous (u.s.c.) map. The chain map

$$
\varphi: C_{*}\left(\tau_{2}\right) \rightarrow C_{*}(\mu)
$$

is called a $\left(\tau_{1}, \mu\right)$-chain approximation (or chain approximation) of the map $F$ (with coefficients in a ring $\mathbb{A}$ ) if for every block $e \in \tau_{2}$ there is a point $s(e) \in K$ such that (a) $e \subset S t\left(s(e), \tau_{1}\right)$, and (b) $|\varphi(e)| \subset S t(F(s(e)), \mu)$.

ReMARK 4. 1. For upper semicontinuous maps see [2].

2. In [25], [26] it is proved that every $\mathbb{F}$-acyclic map $F$ has arbitrarily close chain approximations (with coefficients in $\mathbb{F}$, i.e., for every block structure $\tau_{1}$ and $\mu$ there is a block structure $\tau_{2}, \tau_{2}>\tau_{1}$, and a chain map $\varphi: C_{*}\left(\tau_{2}\right) \rightarrow C_{*}(\mu)$ which is a $\left(\tau_{1}, \mu\right)$-chain approximation of $F$. If $\tau_{1} \in \hat{\tau}$ and $\mu \in \hat{\mu}$ ( $\hat{\tau}$ and $\hat{\mu}$ are f.s.b.d. of $K$ and $L$ respectively) then $\tau_{2} \in \hat{\tau}$. We say $F$ has a chain approximation with respect to $\hat{\tau}$ and $\hat{\mu}$. 
3. If $F: K \rightarrow L$ is an u.s.c. map which has chain approximations with respect to a given f.s.b.d. $\hat{\tau}$ and $\hat{\mu}$ of $K$ and $L$, respectively, then $F$ has chain approximation with respect to any f.s.b.d. $\hat{\tau}_{1}$ and $\hat{\mu}_{1}$ of $K$ and $L$, respectively (follows from [26]).

4. In $[9,22]$ the existence of arbitrary close chain approximations (with coefficients in $\mathbb{F}$ ) for $(1, n)$-maps $F: K \rightarrow L, n \geq 2$, is proved.

Definition 9. Let $K, L$ be finite simplicial complexes with triangulations $\bar{\tau}, \bar{\mu}$, resp., and let $\hat{\tau}=\left\{\tau_{i}\right\}, \hat{\mu}=\left\{\mu_{i}\right\}$ be f.s.b.d. with respect to $\bar{\tau}$ and $\bar{\mu}$, resp. Let $F: K \rightarrow L$ be an u.s.c. map. The graded set

$$
A(F)=\left\{A(F)_{i} \mid i \in \mathbb{N}\right\}
$$

where $A(F)_{i}$ is a nonempty set of chain maps from $C_{*}\left(\tau_{i}\right)$ to $C_{*}\left(\mu_{i}\right)$ with coefficients in a ring $\mathbb{A}$ is called an approximation system (Asystem) of $F$ with respect to $\hat{\tau}$ and $\hat{\mu}$ if

(a) for every $\varphi \in A(F)_{i}$ there is $k>i, k \in \mathbb{N}$, such that $\varphi=$ $\bar{\varphi} b(i, k)$ and $\bar{\varphi}: C_{*}\left(\tau_{k}\right) \rightarrow C_{*}\left(\mu_{i}\right)$ is a $\left(\tau_{i}, \mu_{i}\right)$-chain approximation of $F$, where $b(i, k): C_{*}\left(\tau_{i}\right) \rightarrow C_{*}\left(\tau_{k}\right)$ is a barycentric subdivision, and

(b) for every $i \in \mathbb{N}$ there is $i_{1}>i, i_{1} \in \mathbb{N}$, such that for $m \geq$ $l \geq i_{1}, \varphi=\bar{\varphi} b\left(l, l_{1}\right) \in A(F)_{l}, \psi=\bar{\psi} b\left(m, m_{1}\right) \in A(F)_{m}$ with $m_{1} \geq l_{1}$, the chain maps $\bar{\varphi} \chi\left(m_{1}, l_{1}\right)$ and $\chi(m, l) \bar{\psi}$ are homotopic with a chain homotopy $D$ satisfying the following condition: for every block $e \in \tau_{m_{1}}$ there is a point $b(e) \in K$ such that $e \subset S t\left(b(e), \tau_{i}\right)$ and $|D(e)| \subset S t\left(F(b(e)), \mu_{i}\right)$.

REMARK 5. 1. In [26] it is proved that every $\mathbb{F}$-acyclic map has an A-system (coefficients in the field $\mathbb{F}$ ) with respect to a given f.s.b.d. $\hat{\tau}$ and $\hat{\mu}$ with Kronecker index, $K I A(F)=1$, i.e., for every $\varphi \in A(F)$, $K I \varphi=1$. The Kronecker index $K I \varphi$ of a chain map $\varphi: C_{*}\left(\tau_{i}\right) \rightarrow$ $C_{*}\left(\mu_{i}\right)$ is defined as $K I \varphi=\varepsilon(\varphi(e))$, where $\varepsilon: C_{0}\left(\mu_{i}\right) \rightarrow A$ is the augmentation of the chain complex $C_{*}\left(\mu_{i}\right)$ and $e$ is a 0 -dimensional block, see [27, p. 167]. Since we consider connected polyhedra the Kronecker index $K I \varphi$ of the chain map $\varphi$ is well defined.

2. In [9] the existence of an A-system $A(F)$ for every $(1, n)$-map with $K I A(F)=n$ with coefficients in a fixed field $\mathbb{F}$ is proved. In particular, if one chooses $\mathbb{F}$ to be $\mathbb{Z}_{2}$ and $n$ odd there exists an Asystem of $F$ with Kronecker index different from zero. We consider only A-systems with nonzero Kronecker index.

4. Equivariant maps, block complexes and A-systems. Let $X$ be a compact space. The single valued continuous map $\gamma: X \rightarrow X$ is called 
an involution if $\gamma^{2}(x)=x$ for all $x \in X$. The involution is called free if $\gamma(x) \neq x$ for all $x \in X$. If $\gamma(x)=x$ for all $x \in X$ then $\gamma$ is called the trivial involution. By $\alpha$ we denote the antipodal involution on $\mathbb{R}^{n}, \alpha(x)=-x, x \in \mathbb{R}^{n}$, or some $\alpha$-invariant subset of $\mathbb{R}^{n}$.

Let $X_{i}, i=1,2$, be spaces with involution $\gamma_{i}: X_{i} \rightarrow X_{i}$, resp. and $F: X_{1} \rightarrow X_{2}$ an $m$-acyclic map (w.r.t. $\mathbb{A}$ ) and multiplicity function $m: \widetilde{\Gamma}(F) \rightarrow \mathbb{A}$. Suppose that $F$ is $\left(\gamma_{1}, \gamma_{2}\right)$-equivariant, i.e., $F\left(\gamma_{1}(x)\right)=\gamma_{2}(F(x))$. Then there is an involution $\gamma$ on the graph $\Gamma(F)=\left\{(x, y) \in X_{1} \times X_{2} \mid y \in F(x)\right\}$ defined by $\gamma(x, y)=\left(\gamma_{1}(x)\right.$, $\left.\gamma_{2}(y)\right)$ with $(x, y) \in X_{1} \times X_{2}$. The involution $\gamma$ induces an involution on $\widetilde{\Gamma}(F)$ which we also denote by $\gamma$.

Definition 10. Let $X_{i}, i=1,2$, be spaces with involution $\gamma_{i}$, respectively. The $m$-acyclic map $F: X_{1} \rightarrow X_{2}$ w.r.t. $\mathbb{A}$ with multiplicity function $m: \widetilde{\Gamma}(F) \rightarrow \mathbb{A}$ is called $\left(\gamma_{1}, \gamma_{2}\right)$-equivariant if

(a) $F\left(\gamma_{1}(x)\right)=\gamma_{2}(F(x))$ for all $x \in X_{1}$, and

(b) $m(\gamma(x, C(x)))=m(x, C(x))$ for all $(x, C(x)) \in \widetilde{\Gamma}(F)$.

Let $K$ be a simplicial complex with a block structure $\tau_{1}$ and involution $\gamma: K \rightarrow K$. The block structure $\tau_{1}$ is $\gamma$-equivariant if $\gamma(e)$ is a block in $\tau_{1}$ for every block $e \in \tau_{1}$.

REMARK 6. 1. The map $F: X_{1} \rightarrow X_{2}$ is called $\left(\gamma_{1}, \gamma_{2}\right)$-equivariant if the condition (a) of Definition 10 is fullfilled. If $\hat{\tau}$ is a f.s.b.d. on $K, \hat{\tau}$ is called $\gamma$-equivariant if each $\tau_{i}$ is $\gamma$-equivariant.

2. Let $\mathbb{R}^{m}=\left\{\left(x_{1}, \ldots, x_{m}\right) \mid x_{i} \in \mathbb{R}\right\}$ be the $m$-dimensional Euclidean space. If $n>m$ we consider $\mathbb{R}^{m}$ as a linear subspace of $\mathbb{R}^{n}$ consisting of the points $\left(x_{1}, \ldots, x_{m}, 0, \ldots, 0\right)$.

Let $a \in \mathbb{R}, a>0$, then $D_{a}^{m}$ denotes the cube

$$
D_{a}^{m}=\left\{\left(x_{1}, \ldots, x_{m}\right)|| x_{i} \mid \leq a\right\} .
$$

The inclusion $l: \mathbb{R}^{m} \rightarrow \mathbb{R}^{n}(n>m)$ induces the inclusion $D_{a}^{m} \subset D_{a}^{n}$. The boundary of $D_{a}^{n+1}$ is denoted by $S_{a}^{n}$, or simply $S^{n}$, again $S^{m} \subset$ $S^{n}$ for $n>m$. The antipodal involution $\alpha$ acts on both spaces $D_{a}^{n}$ and $S_{a}^{n}$.

We consider the following block structure $\mu(a)$ (cubic block subdivision of $\mathbb{R}^{n}$ ) which consists of all translations of $D_{a}^{n}$ with vectors $\sum_{i=1}^{n} 2 a k_{i} e_{i}$, where $e_{i}$ denotes the $i$-th unit vector and $k_{i} \in \mathbb{Z}$. If $a \in \mathbb{N}$ then $\mu_{k}=\mu(1 / k), k \in \mathbb{N}$, is a block structure on $D_{a}^{n}$, and $S_{a}^{n}$ is a block subcomplex. $S_{a}^{m}$ is a block subcomplex in $S_{a}^{n}, n>m$, also. The block structure on $S_{a}^{n}$ induced by $\mu_{k}$ is denoted by $\tau_{k}$. Then $\hat{\mu}=\left\{\mu_{k}\right\}$ and $\hat{\tau}=\left\{\tau_{k}\right\}$ are $\alpha$-equivariant f.s.b.d. of $D_{a}^{n}$ and $S_{a}^{n}$, respectively. 
3. Let $K$ be a block complex with a given f.s.b.d. $\hat{\tau}=\left\{\tau_{i}\right\}$. The simplicial complex $K \times K$ has a natural block structure $\tau_{i} \times \tau_{i}$ induced by $\tau_{i}$ for every $i \in \mathbb{N}$ :

$$
\tau_{i} \times \tau_{i}=\left\{e_{1} \times e_{2} \mid e_{i} \in \tau_{i}, \quad i=1,2\right\} .
$$

Then $\hat{\tau} \times \hat{\tau}$ is a f.s.b.d. for $K \times K$. We call $\hat{\tau} \times \hat{\tau}$ the product block structure. E.g., the block structure $\hat{\mu}=\left\{\mu_{k}\right\}$ of $D_{a}^{n}$ is a product of block structures on the interval $D_{a}^{1}$.

4. On the space $K \times K$ acts the involution $T: K \times K \rightarrow K \times K$, $T(x, y)=(y, x)$. The f.s.b.d. $\hat{\tau} \times \hat{\tau}$ is $T$-equivariant.

Let $K_{s}, s=1,2$, be finite simplicial complexes with involutions $\gamma_{s}$ and $\gamma_{s}$-equivariant f.s.b.d. $\hat{\tau}_{s}=\left\{\tau_{i}^{s}\right\}$. Since the block structures $\tau_{i}^{s}$ are $\gamma_{s}$-equivariant each $\gamma_{s}$ induces a chain map which we denote also by $\gamma_{s}$ :

$$
\gamma_{s}: C_{*}\left(\tau_{i}^{s}\right) \rightarrow C_{*}\left(\tau_{i}^{s}\right) .
$$

A chain map (or homomorphism) $\varphi: C_{*}\left(\tau_{i}^{1}\right) \rightarrow C_{*}\left(\tau_{i}^{2}\right)$ is $\left(\gamma_{1}, \gamma_{2}\right)$ equivariant if $\varphi \gamma_{1}=\gamma_{2} \varphi$. If $K_{1}=K_{2}, \gamma_{1}=\gamma_{2}=\gamma$ we simply say $\gamma$-equivariant.

Since the f.s.b.d. $\hat{\tau}_{s}$ are $\gamma_{s}$-equivariant the chain subdivision maps $b(k+1, k)=b\left(\tau_{k}^{s}, \tau_{k+1}^{s}\right)$ are $\gamma_{s}$-equivariant too. In this situation we choose chain approximations of the identity $\chi(k+1, k)=\chi\left(\tau_{k+1}^{s}, \tau_{k}^{s}\right)$ to be $\gamma_{s}$-equivariant also, and we consider $C_{*}\left(\tau^{s}\right)$ with these chain maps.

Let $F: K_{1} \rightarrow K_{2}$ be a $\left(\gamma_{1}, \gamma_{2}\right)$-equivariant u.s.c. map. The Asystem, $A(F)$, with respect to a $\gamma_{s}$-equivariant f.s.b.d. $\hat{\tau}_{s}, s=1,2$, is called $\left(\gamma_{1}, \gamma_{2}\right)$-equivariant if every chain map $\varphi \in A(F)$ is $\left(\gamma_{1}, \gamma_{2}\right)$ equivariant. Again, if $K_{1}=K_{2}, \gamma_{1}=\gamma_{2}=\gamma$ the A-system $A(F)$ is called $\gamma$-equivariant.

Definition 11 ([29, p. 60]). Let $\left(K_{1}, \tau_{1}\right)$ and $\left(K_{2}, \tau_{2}\right)$ be block complexes. A carrier from $\left(K_{1}, \tau_{1}\right)$ to $\left(K_{2}, \tau_{2}\right)$ is a function $\mathscr{C}$ which assigns to each block $e \in \tau_{1}$ a block subcomplex $\mathscr{C}(e)$ in $\tau_{2}$ such that for $e_{1} \in e, \mathscr{C}\left(e_{1}\right) \subset \mathscr{C}(e)$. An $n$-acyclic (resp. acyclic) carrier $\mathscr{C}$ satisfies $\widetilde{H}_{i}(\mathscr{C}(e))=0$ for $0 \leq i \leq n$ (resp. for all $i$ ) and every block $e \in \tau_{1}$ (coefficients in $\mathbb{A}$ ).

If $\gamma_{i}$ is an involution on $K_{i}$ such that the block structure $\tau_{i}$ is $\gamma_{i}$-equivariant, $i=1,2$. Then the carrier $\mathscr{C}$ is called $\left(\gamma_{1}, \gamma_{2}\right)$ equivariant (or equivariant) if $\mathscr{C}\left(\gamma_{1}(e)\right)=\gamma_{2}(\mathscr{C}(e))$ for all blocks $e \in \tau_{1}$. A chain map $\varphi: C_{*}\left(\tau_{1}\right) \rightarrow C_{*}\left(\tau_{2}\right)$ is carried by the carrier $\mathscr{C}$ if $\varphi(e)$ is a chain in the complex $\mathscr{C}(e)$ for every block $e \in \tau_{1}$. 
A map $f: K_{1} \rightarrow K_{2}$ is carried by the carrier $\mathscr{C}$ if $f(e) \subset \mathscr{C}(e)$ for every block $e \in \tau_{1}$.

REMARK 7. 1. The block structure $\hat{\tau}=\left\{\tau_{k}\right\}$ on $S_{a}^{n}$ is $\alpha$-equivariant and the block complex $S_{a}^{n}$ is $\alpha$-free cell complex, i.e., $\alpha(e) \cap e=\varnothing$ for every $e \in \tau_{k}$

2. For later use we note the following. The covering $w_{1}=\left\{A_{1}, \ldots\right.$, $\left.A_{N}\right\}$ of a given space is finer than the covering $w_{2}=\left\{B_{1}, \ldots, B_{M}\right\}$ of the same space if every $A_{i}$ is contained in some $B_{j}$. If the covering $w_{1}$ is finer than the covering $w_{2}$ we denote this by $w_{1}>w_{2}$.

II. Borsuk, Borsuk-Ulam and Bourgin-Yang theorems for chain mappings. In this section we state some lemmata which will be important for the following. In this section we consider chains, A-systems, and homology with $\mathbb{Z}_{2}$ coefficients.

For a block complex $(K, \tau)$ we denote by $K^{(i)}$ the $i$-dimensional skeleton of $K$ w.r.t. $\tau$.

LEMMA 1. Let $S^{n}, n \geq 1$, be the $n$-dimensional sphere with the $\alpha$-equivariant block structure $\tau_{k}$. Then there exists an $\alpha$-equivariant chain map

$$
\beta: C_{*}\left(\left(S^{n}\right)^{(n-1)}, \tau_{k}\right) \rightarrow C_{*}\left(S^{n-1}, \tau_{k}\right)
$$

with Kronecker index $K I \beta \equiv 1(\bmod 2)$ and such that

(a) the chain map $\beta$ with values in $C_{*}\left(S^{n}, \tau_{k}\right)$ is chain homotopic to the identity inclusion $\left(S^{n}\right)^{n-1} \subset S^{n}$ with an $\alpha$-equivariant chain homotopy $D$, and

(b) for each block $e$ in $S^{n-1}$ one has $\beta(e)=e$ and $D(e)=0$.

Proof. Consider the carrier $\mathscr{C}:\left(\left(S^{n}\right)^{(n-1)}, \tau_{k}\right) \rightarrow\left(S^{n-1}, \tau_{k}\right)$, defined by $\mathscr{C}(e)=S^{n-1}$ for every block $e \in\left(S^{n}\right)^{(n-1)}$, cf. Definition I.11. $\mathscr{C}$ is an $\alpha$-equivariant $(n-2)$-acyclic carrier. Since $\left(\left(S^{n}\right)^{n-1}, \tau_{k}\right)$ is a free $\alpha$-complex, Lemma 2.2 in [29] implies the existence of an $\alpha$-equivariant extension $\beta$ of the identity chain map on $S^{n-1}$ over $\left(S^{n}\right)^{(n-1)}$. Furthermore, $\beta$ considered as a chain map with values in $C_{*}\left(S^{n}, \tau_{k}\right)$ and the chain map induced by the identity inclusion of $\left(S^{n}\right)^{(n-1)} \subset S^{n}$ are chain homotopic with equivariant chain homotopy $D$ satisfying (b).

REMARK 1. Lemma 2.2 in [29] is proved for an acyclic carrier, but for our purposes it suffices to assume that $\mathscr{C}$ is an $(n-2)$-acyclic carrier. 
The following lemma shows that each $\alpha$-equivariant chain map $\kappa: C^{*}\left(S^{n}, \tau_{k}\right) \rightarrow C^{*}\left(S^{n}, \tau_{k}\right)$ is homotopic to an $\alpha$-equivariant map $\bar{\kappa}$, which is a chain map on $\left(S^{n-1}, \tau_{k}\right)$.

LEMMA 2. Let $\kappa: C^{*}\left(S^{n}, \tau_{k}\right) \rightarrow C^{*}\left(S^{n}, \tau_{k}\right)$ be an $\alpha$-equivariant chain map. Then there exists an $\alpha$-equivariant chain map $\bar{\kappa}$ : $C^{*}\left(S^{n}, \tau_{k}\right) \rightarrow C^{*}\left(S^{n}, \tau_{k}\right)$ such that

(a) $\kappa$ and $\bar{\kappa}$ are chain homotopic, and

(b) for all blocks $e \in S^{n-1}, \bar{\kappa}(e)$ is a chain in $S^{n-1}$.

Proof. Let $\beta=\left\{\beta_{i}\right\}, D=\left\{D_{i}\right\}, i=1,2$, be as in Lemma 1 and let $e$ be an $i$-dimensional block in $S^{n}$ with $\partial e=\sum_{j} e(j)$. Define

$$
\bar{\kappa}_{i}(e)= \begin{cases}\beta_{i}(\kappa(e)) & \text { for } e \in S^{n-1}, \\ \kappa(e)+D_{i-1}(\kappa(e(j))) & \text { if } e \cap S^{n-1}=e(j), \\ \kappa(e) & \text { otherwise. }\end{cases}
$$

Then $\bar{\kappa}=\left\{\bar{\kappa}_{i} \mid i=0, \ldots, n\right\}$ is an $\alpha$-equivariant chain map satisfying (b) and $K I \kappa=K I \bar{\kappa} \equiv 1(\bmod 2)$. Consider the carrier $\mathscr{C}:\left(S^{n}, \tau_{k}\right) \rightarrow$ $\left(S^{n}, \tau_{k}\right), \mathscr{C}(e)=S^{n}$ for every block $e \in \tau_{k}$. It is an $(n-1)$-acyclic carrier. The chain maps $\kappa$ and $\bar{\kappa}$ are carried by $\mathscr{C}$ (cf. Definition I.11). Since $\tau_{k}$ is an $\alpha$-free complex and the Kronecker indices of these chain maps are equal, $K I \kappa=K I \bar{\kappa} \equiv 1(\bmod 2)$, using Remark 1 following Lemma 2.2 in [29], we conclude that the chain maps $\kappa$ and $\bar{\kappa}$ are homotopic.

LemMA 3. Let $\kappa: C_{*}\left(S^{n}, \tau_{k}\right) \rightarrow C_{*}\left(S^{n}, \tau_{k}\right)$ be an $\alpha$-equivariant chain map with Kronecker index $K I \kappa \equiv 1(\bmod 2)$. Then $\operatorname{deg} \kappa \equiv 1$ $(\bmod 2)$, where deg denotes the degree of $\kappa$.

Proof. Due to Lemma 2 we may assume that $\kappa: C_{*}\left(S^{n-1}, \tau_{k}\right) \rightarrow$ $C_{*}\left(S^{n-1}, \tau_{k}\right)$. Because chain homotopy does not change either the Kronecker index or the degree this is no restriction. The proof proceeds by induction on $n$. We start with $n=1$.

Due to our assumption $\kappa: C_{*}\left(S^{0}, \tau_{k}\right) \rightarrow C_{*}\left(S^{0}, \tau_{k}\right)$ and since $K I \kappa$ $\equiv 1(\bmod 2)$ we obtain $\kappa(1)=1$ or $\kappa(1)=\overline{1}, S^{0}=\{1, \overline{1}\}$. Let $D_{+}=\left\{\left(x_{1}, x_{2}\right) \in S^{1} \mid x_{2} \geq 0\right\}, D_{-}=\left\{\left(x_{1}, x_{2}\right) \in S^{1} \mid x_{2} \leq 0\right\}$ be subcomplexes of $S^{1}$ which we consider as 1-dimensional chains in $\tau_{k}$, such that $\partial D_{+}=\overline{1}+1=\partial D_{-}$(coefficients in $\left.\mathbb{Z}_{2}\right)$. The chain $\kappa\left(D_{+}\right)$ may be written as $\kappa\left(D_{+}\right)=u D_{+}+v D_{-}$and since $\kappa(1)=1$ or $\overline{1}$, then $\partial \kappa\left(D_{+}\right)=(u+v)(1+\overline{1})=1+\overline{1}(\bmod 2)$. Now, $(u+v) \equiv 1(\bmod 2)$ yields 


$$
\operatorname{deg} \kappa\left(D_{+}+D_{-}\right)=\kappa\left(D_{+}+D_{-}\right) \equiv D_{+}+D_{-} \quad(\bmod 2),
$$

therefore $\operatorname{deg} \kappa \equiv 1(\bmod 2)$.

Now let us assume that the assumption is true up to $n-1$. Then $\left.\kappa\right|_{S^{n-1}}: C_{*}\left(S^{n-1}, \tau_{k}\right) \rightarrow C_{*}\left(S^{n-1}, \tau_{k}\right)$ is $\alpha$-equivariant and $\operatorname{deg}\left(\left.\kappa\right|_{S^{n-1}}\right)$ $\equiv 1(\bmod 2)$. Let

$$
D_{+}=\left\{\left(x_{1}, \ldots, x_{n+1}\right) \mid x_{n+1} \geq 0\right\}, \quad D_{-}=\left\{\left(x_{1}, \ldots, x_{n+1}\right) \mid x_{n+1} \leq 0\right\}
$$

be considered as chains in $S^{n}$. If we consider $S^{n-1}$ as an $(n-1)$ dimensional chain in $\tau_{k}$ we obtain the relation $\partial D_{+}=\partial D_{-}=S^{n-1}$ (w.r.t. $\mathbb{Z}_{2}$ coefficients).

Now we calculate $\kappa\left(D_{+}+D_{-}\right)$using $\kappa\left(D_{+}\right)=u D_{+}+v D_{-}$, $\left.\operatorname{deg} \kappa\right|_{S^{n-1}}\left(S^{n-1}\right)=\kappa\left(\partial D_{+}\right)=(u+v)\left(S^{n-1}\right)=S^{n-1}$ and $u+v=$ $\operatorname{deg}\left(\left.\kappa\right|_{S^{n-1}}\right) \equiv(\bmod 2)$. Then

$$
\operatorname{deg} \kappa\left(D_{+}+D_{-}\right)=\kappa\left(D_{+}+D_{-}\right)=(u+v)\left(D_{+}+D_{-}\right)=D_{+}+D_{-} .
$$

Therefore $\operatorname{deg} \kappa \equiv 1(\bmod 2)$.

An immediate consequence is

CoRollary 4 (Borsuk theorem for chain maps). Let $\kappa: C_{*}\left(S^{n} \tau_{k}\right)$ $\rightarrow C_{*}\left(S^{m}, \tau_{k}\right)$ be an $\alpha$-equivariant chain map with Kronecker index $K I \kappa \equiv 1(\bmod 2)$. Then $m \geq n$.

LEMMA 5 (Borsuk-Ulam theorem for chain maps). Let $\kappa: C_{*}\left(S^{n}, \tau\right)$ $\rightarrow C_{*}\left(D_{s}^{n}, \mu_{k}\right)$ (cf. Remark I.6.2) be a chain map with Kronecker index $K I \kappa \equiv 1(\bmod 2)$. Then there exists a block $e \in \tau$ such that $|\kappa(e)| \cap|\kappa(\alpha(e))| \neq \varnothing$.

Proof. Assume that

$$
|\kappa(e)| \cap|\kappa(\alpha(e))|=\varnothing \quad \text { for all blocks } e \in \tau .
$$

Consider the map $\bar{\Delta}: S^{n} \rightarrow S^{n} \times S^{n}$ defined by $\bar{\Delta}(x)=(x,-x)$ and the block structure on $S^{n} \times S^{n}$ given by $\tau \times \tau$. The map $\bar{\Delta}$ is $(\alpha, T)$ equivariant (cf. I.4, and Remark I.6.2). The carrier $\mathscr{C}:\left(S^{n}, \tau\right) \rightarrow$ $\left(S^{n} \times S^{n}, \tau \times \tau\right)$, with $\mathscr{C}(e)=e \times \alpha(e)$ is $(\alpha, T)$-invariant and acyclic $(\bar{\Delta}$ is carried by $\mathscr{C})$. Since $\left(S^{n}, \tau\right)$ is an $\alpha$-free block complex, Lemma 2.2 in [29] implies the existence of an $(\alpha, T)$-equivariant chain map $\bar{\Delta}_{\sharp}: C_{*}\left(S^{n}, \tau\right) \rightarrow C_{*}\left(S^{n} \times S^{n}, \tau \times \tau\right)$ which is carried by the carrier $\mathscr{C}$, i.e., $\bar{\Delta}_{\#}(e)$ is a chain in $e \times \alpha(e)$ for all blocks in $\tau$ and $K I \bar{\Delta}_{\#} \equiv 1(\bmod 2)$. 
Consider the chain map $(\kappa \otimes \kappa) \bar{\Delta}_{\#}: C_{*}(\tau) \rightarrow C_{*}(\tau \times \tau)$. Since $\left|\bar{\Delta}_{\#}(e)\right| \subset e \times \alpha(e)$ for every block $e \in \tau$ then

$$
\left|(\kappa \otimes \kappa) \bar{\Delta}_{\#}(e)\right| \subset|\kappa(e)| \times|\kappa(\alpha(e))|
$$

and from (1) we obtain

$$
\left|(\kappa \otimes \kappa) \bar{\Delta}_{\#}(e)\right| \cap\left|(\kappa \otimes \kappa) \bar{\Delta}_{\#}(\alpha(e))\right|=\varnothing
$$

for every block $e \in \tau$.

The chain map $(\kappa \otimes \kappa) \bar{\Delta}_{\#}$ is $(\alpha, T)$-equivariant and from (2) it follows that

$$
\left|(\kappa \otimes \kappa) \bar{\Delta}_{\#}(e)\right| \cap M=\varnothing
$$

for every block $e \in \tau$ and $M=\left\{(x, y) \in D_{s}^{n} \times D_{s}^{n} \mid x=y\right\}$. Then there exists a block subdivision $\mu_{l}(l>k)$ of the block structure $\mu_{k}$ (cf. Definition I.6) such that $\left|(\kappa \otimes \kappa) \bar{\Delta}_{\#}(e)\right| \cap M^{\prime}=\varnothing$ for every block $e$ in $\tau$, and $M^{\prime}=S t\left(M, \mu_{l} \times \mu_{l}\right)$. Let $N$ be the closure of $D_{s}^{n} \times D_{s}^{n} \backslash M^{\prime}$. Then $N$ is a subcomplex in $D_{s}^{n} \times D_{s}^{n}$ with respect to the block structure $\mu_{l} \times \mu_{l}$ and $N$ is $T$-equivariant. Since $\left|(\kappa \otimes \kappa) \bar{\Delta}_{\#}(e)\right|$ is in $N$ then $b\left(\mu_{k} \times \mu_{k}, \mu_{l} \times \mu_{l}\right)(\kappa \otimes \kappa) \bar{\Delta}_{\#}(e)$ is a chain in $N$ and therefore $b\left(\mu_{k} \times \mu_{k}, \mu_{l} \times \mu_{l}\right)(\kappa \otimes \kappa) \bar{\Delta}_{\#}$ is a chain map from $C_{*}\left(S^{n}, \tau\right)$ to $C_{*}\left(N, \mu_{l} \times \mu_{l}\right)$ which is $(\alpha, T)$-equivariant (cf. I.2 for the chain subdivision $b$ ).

Let $d: N \rightarrow S^{n-1}$ be defined by $d(u, v)=(u-v) /\|u-v\|$. Obviously $d$ is $(\alpha, T)$-equivariant. Let $d_{\#}: C_{*}\left(N, \mu_{l} \times \mu_{l}\right) \rightarrow C_{*}\left(S^{n-1}, \tau\right)$ be the $(\alpha, T)$-equivariant chain map induced by $d$ with Kronecker index $K I d_{\#} \equiv 1(\bmod 2)$ for $l$ sufficiently large.

Finally, consider the chain map

$$
\Xi=d_{\#} b\left(\mu_{k} \times \mu_{k}, \mu_{l} \times \mu_{l}\right)(\kappa \otimes \kappa) \bar{\Delta}: C_{*}\left(S^{n}, \tau\right) \rightarrow C_{*}\left(S^{n-1}, \tau\right),
$$

which is $\alpha$-equivariant with Kronecker index $K I \Xi \equiv 1(\bmod 2)$. This contradicts Corollary 4.

Let $(K, \nu)$ be a block complex with $\gamma$-equivariant block structure $\nu$ and $\gamma$ a free involution on $K$.

Definition 6. The chain genus of a $\gamma$-free block complex $(K, \nu)$ is defined by

$$
g_{c}(K, \gamma)=\min \left\{l \begin{array}{l}
\begin{array}{l}
\text { there exists a }(\gamma, \alpha) \text {-equivariant } \\
\text { chain map } \eta: C_{*}(K, \nu) \rightarrow C_{*}\left(S^{l}, \tau_{k}\right) \\
\text { with } K I \eta \equiv 1(\bmod 2) \text { and }|\eta(e)| \cap \\
|\eta(\alpha(e))|=\varnothing \text { for all } e \in \nu .
\end{array}
\end{array}\right\} .
$$


REMARK 2. The genus $g(K, \gamma)$ of a free involution $\gamma$ on $K$ is defined as

$$
g(K, \gamma)=\min \left\{l \mid \begin{array}{l}
\text { there is a }(\gamma, \alpha) \text {-equivariant, continuous, } \\
\text { single valued map } f: K \rightarrow S^{l} ;
\end{array}\right\}
$$

it is easy to see that $g(K, \gamma) \geq g_{c}(K, \gamma)$. Furthermore, recall that $\operatorname{dim} K \geq g(K, \gamma)$, cf. [30]. Therefore $\operatorname{dim} K \geq g_{c}(K, \gamma)$.

CorollaRy 7. Let $\kappa: C_{*}\left(S^{n+k}, \tau_{l}\right) \rightarrow C_{*}\left(D^{n}, \mu_{l}\right), k \geq 0$, be $a$ chain map with Kronecker index $K I \kappa \equiv 1(\bmod 2)$. Then $g_{c}(B(\kappa), \gamma)$ $\geq k$, with $B(\kappa)=\bigcup\left\{e \in \tau_{k}|| \kappa(e)|\cap| \kappa(\alpha(e)) \mid \neq \varnothing\right\}$.

Proof. The case $k=0$ is an immediate consequence of Lemma 5. We assume $k \geq 1$ and use the idea in [12, 2.6]. Assume that $g_{c}(B(\kappa), \alpha)=m<k$. Then we consider the chain map $\eta$ : $C_{*}\left(B(\kappa), \tau_{l}\right) \rightarrow C_{*}\left(S^{m}, \tau_{l}\right)$ (cf. Definition 6) as a chain map in $C_{*}\left(D^{m+1}, \mu_{l}\right) \quad\left(S^{m}\right.$ is a boundary of $\left.D^{m+1}\right)$. Let $\mathscr{C}$ be the carrier from $B(\kappa)$ in $D^{m+1}$ defined by $\mathscr{C}(e)=D^{m+1}$ for all blocks. This is an $\alpha$-equivariant acyclic carrier. Since $K I \eta \equiv 1(\bmod 2)$ there is an ( $\alpha$-equivariant) chain map

$$
\tilde{\eta}: C_{*}\left(S^{n+k}, \tau_{l}\right) \rightarrow C_{*}\left(D^{m+1}, \mu_{l}\right)
$$

with Kronecker index $K I \tilde{\eta} \equiv 1(\bmod 2)$ and which is an extension of the chain map $\eta$ (Lemma 2.2, [29]).

Consider the carrier $\mathscr{C}_{1}$ from $\left(S^{n+k}, \tau_{l}\right)$ in $\left(S^{n+k} \times S^{n+k}, \tau_{l} \times \tau_{l}\right)$ defined by $\mathscr{C}_{1}(e)=e \times e$. This is an $(\alpha, \bar{\alpha})$-equivariant acyclic carrier, with $\bar{\alpha}(x, y)=(\alpha(x), \alpha(y))$ for all $(x, y) \in S^{n+k} \times S^{n+k}$. Lemma 2.2 in [29] guarantees the existence of an $(\alpha, \bar{\alpha})$-equivariant chain map $\Delta_{\#}: C_{*}\left(\tau_{l}\right) \rightarrow C_{*}\left(\tau_{l} \times \tau_{l}\right)$ with $K I \Delta_{\#} \equiv 1(\bmod 2)$ and $\left|\Delta_{\#}(e)\right| \subset \mathscr{C}_{1}(e)$ for all blocks $e \in \tau_{l}$.

Since $C_{*}\left(D^{n}, \mu_{l}\right) \otimes C_{*}\left(D^{m+1}, \mu_{l}\right) \approx C_{*}\left(D^{n} \times D^{m+1}, \mu_{l} \times \mu_{l}\right)=$ $C_{*}\left(D^{m+n+1}, \mu_{l}\right)$ we consider the chain map $\Xi=(\kappa \otimes \tilde{\eta}) \Delta_{\#}$ as a chain map

$$
\Xi: C_{*}\left(S^{n+k}, \tau_{l}\right) \rightarrow C_{*}\left(D^{m+n+1}, \mu_{l}\right)
$$

with Kronecker index $K I \Xi \equiv 1(\bmod 2)$. Since $m<k$, then $\left(D^{m+n+1}, \mu_{l}\right) \subset\left(D^{n+k}, \mu_{l}\right)$ and we consider $\Xi$ as a chain map in $C_{*}\left(D^{n+k}, \mu_{l}\right)$.

Then from Lemma 5 there is a block $e \in \tau_{l}$ such that

$$
\left|(\kappa \otimes \tilde{\eta}) \Delta_{\#}(e)\right| \cap\left|(\kappa \otimes \tilde{\eta}) \Delta_{\#}(\alpha(e))\right| \neq \varnothing
$$


and therefore also

$$
(|\kappa(e)| \times|\tilde{\eta}(e)|) \cap(|\kappa(\alpha(e))| \times|\tilde{\eta}(\alpha(e))|) \neq \varnothing,
$$

i.e., $|\kappa(e)| \cap|\kappa(\alpha(e))| \neq \varnothing$ and $|\tilde{\eta}(e)| \cap|\tilde{\eta}(\alpha(e))| \neq \varnothing$. The first implies that $e \in B(\kappa)$, therefore $\tilde{\eta}(e)=\eta(e)$ and $\tilde{\eta}(\alpha(e))=\eta(\alpha(e))$. This is impossible since $|\eta(e)| \cap|\eta(\alpha(e))|=\varnothing$, cf. Definition 6.

COROLlARY 8 (Bourgin-Yang theorem for chain maps). Let $\kappa$ : $C_{*}\left(S^{n+k}, \tau_{l}\right) \rightarrow C_{*}\left(D^{n}, \mu_{l}\right), k \geq 0$, be a chain map with Kronecker index $K I \kappa \equiv 1(\bmod 2)$, then $\operatorname{dim} B(\kappa) \geq k$.

The proof is a direct consequence from Corollary 7 and Remark 2 .

III. Borsuk, Borsuk-Ulam, and Bourgin-Yang theorems for maps with A-systems with coefficients in $\mathbb{Z}_{2}$.

1. Borsuk theorem.

Proposition 1. Let $F:\left(S^{n}, \alpha\right) \rightarrow\left(S^{m}, \alpha\right)$ be an u.s.c. $\alpha$-equivariant map. If $F$ has an $\alpha$-equivariant $A$-system $A(F)$ with coefficients in $\mathbb{Z}_{2}$ then $m \geq n$.

Proof. Let $\varphi \in A(F)_{k}$. Then $\varphi$ is $\alpha$-equivariant and $K I \varphi \equiv$ $1(\bmod 2)$. From Corollary II.4 follows $m \geq n$.

2. Existence of A-systems for products of two maps with A-system. To prove the Borsuk-Ulam theorem for u.s.c. maps $F: S^{n} \rightarrow \mathbb{R}^{n}$ with A-systems (with coefficients in $\mathbb{Z}_{2}$ ) we have to establish the existence of a nontrivial A-system for a product of two maps with A-systems with coefficients in a field $\mathbb{F}$ (cf. Definition I.9).

This will be done first. Let $\left(K_{i}, \hat{\tau}^{i}\right)$ and $\left(L_{i}, \hat{\mu}^{i}\right), i=1,2$, be finite block complexes with fixed f.s.b.d. $\hat{\tau}^{i}, \hat{\mu}^{i}$, respectively (cf. Definition I.7). We consider the product f.s.b.d.

$$
\theta=\hat{\tau}^{1} \times \hat{\tau}^{2}=\left\{\theta_{k}=\tau_{k}^{1} \times \tau_{k}^{2} \mid k=0,1, \ldots\right\}
$$

on $K_{1} \times K_{2}$, and

$$
\bar{\theta}=\hat{\mu}^{1} \times \hat{\mu}^{2}=\left\{\bar{\theta}_{k}=\mu_{k}^{1} \times \mu_{k}^{2} \mid k=0,1, \ldots\right\}
$$

on $L_{1} \times L_{2}$, respectively (cf. Remark I.6.3).

The tensor product $C_{*}=C_{*}\left(K_{1}, \tau_{k}^{1}\right) \otimes C_{*}\left(K_{2}, \tau_{k}^{2}\right)$ (over the field $\mathbb{F}$ ) is a chain complex with differential $\partial\left(e_{1} \otimes e_{2}\right)=\partial e_{1} \otimes e_{2}+(-1)^{\operatorname{dim} e_{1}} e_{1} \otimes$ $\partial e_{2}, e_{j} \in \tau_{k}^{j}$, cf. [8, 9.1, p. 161]. Using [8, 9.21(4), p. 166] we may 
identify the chain complexes $C_{*}\left(K_{1} \times K_{2}, \theta_{k}\right)$ and $C_{*}$ via the chain isomorphism defined on the generators by $e_{1} \times e_{2} \mapsto e_{1} \otimes e_{2}$.

Following [26], we consider the chain subdivision maps

$$
b_{i}(k, l): C_{*}\left(K_{i}, \tau_{k}^{k}\right) \rightarrow C_{*}\left(K_{i}, \tau_{l}^{i}\right)
$$

and a chain approximation of the identity (see I.2) for $l>k$

$$
\chi_{i}(l, k): C_{*}\left(K_{i}, \tau_{l}^{i}\right) \rightarrow C_{*}\left(K_{i}, \tau_{k}^{i}\right) .
$$

For the chain complex $C_{*}\left(K_{1} \times K_{2}, \theta\right)$ we consider analogously the chain maps $b(k, l)$ and $\chi(l, k)$ defined by

$$
\begin{aligned}
& b(k, l)=b_{1}(k, l) \otimes b_{2}(k, l), \\
& \chi(l, k)=\chi_{1}(l, k) \otimes \chi_{2}(l, k)
\end{aligned}
$$

which gives the chain subdivision and a chain approximation of the identity map for a f.s.b.d. $\theta$ on $K_{1} \times K_{2}$. We call it the tensor product subdivision and a tensor product approximation of the identity.

For the block complexes $\left(L_{i}, \hat{\mu}^{i}=\left\{\mu_{k}^{i}\right\}\right), i=1,2$, we consider $C_{*}\left(L_{1} \times L_{2}, \bar{\theta}\right)$ with the tensor product subdivision $\bar{b}(k, l)$ and a tensor product approximation of the identity $\bar{\chi}(l, k)$.

LEMMA 2. Let $\left(K_{i}, \hat{\tau}^{k}\right),\left(L_{i}, \hat{\mu}^{i}\right)$ be block complexes with f.s.b.d. and let $F_{i}: K_{i} \rightarrow L_{i}$ be u.s.c. maps with A-systems $A\left(F_{i}\right)$ with coefficients in $\mathbb{F}, i=1,2$. Then there exists an A-system with coefficients in $\mathbb{F}$ for the U.s.c. map $F_{1} \times F_{2}: K_{1} \times K_{2} \rightarrow L_{1} \times L_{2}$ with respect to the product f.s.b.d. $\theta=\hat{\tau}^{1} \times \hat{\tau}^{2}, \bar{\theta}=\hat{\mu}^{1} \times \hat{\mu}^{2}$ and with Kronecker index $\left(K I A\left(F_{1}\right)\right)\left(K I A\left(F_{2}\right)\right)$.

Proof. The map $F_{1} \times F_{2}$ is defined by $\left(F_{1} \times F_{2}\right)(x, y)=F_{1}(x) \times F_{2}(y)$ for $(x, y) \in K_{1} \times K_{2}$. Define

$$
A\left(F_{1}\right)_{k} \otimes A\left(F_{2}\right)_{k}=\left\{\varphi_{1} \otimes \varphi_{2} \mid \varphi_{1} \in A\left(F_{1}\right)_{k}, \varphi_{2} \in A\left(F_{2}\right)_{k}\right\} .
$$

The tensor product of $\varphi_{1}$ and $\varphi_{2}$

$$
\varphi_{1} \otimes \varphi_{2}: C_{*}\left(\tau_{k}^{1}\right) \otimes C_{*}\left(\tau_{k}^{2}\right) \rightarrow C_{*}\left(\mu_{k}^{1}\right) \otimes C_{*}\left(\mu_{k}^{2}\right)
$$

is a chain map, cf. [8, p. 162]. We shall show that $A\left(F_{1}\right) \otimes A\left(F_{2}\right)=$ $\left\{A\left(F_{1}\right)_{k} \otimes A\left(F_{2}\right)_{k} \mid k \in \mathbb{N}\right\}$ is an A-system for the u.s.c. map $F_{1} \times F_{2}$ with respect to the f.s.b.d. $\theta$ and $\bar{\theta}$.

Let $k \in \mathbb{N}$ and $\varphi_{1} \otimes \varphi_{2} \in A\left(F_{1}\right)_{k} \otimes A\left(F_{2}\right)_{k}$. Since $\varphi_{i} \in A\left(F_{i}\right)_{k}$, $i=1,2$, there exists an integer $l>k$ (it is no restriction to assume that $l$ is the same for $\varphi_{1}$ and $\left.\varphi_{2}\right)$ such that $\varphi_{i}=\bar{\varphi}_{i} b_{i}(k, l)$ and the chain maps $\bar{\varphi}_{i}$ are $\left(\tau_{k}^{i}, \mu_{k}^{i}\right)$-chain approximations of $F_{i}$, respectively, 
cf. Definition I.9. Writing $\varphi_{1} \otimes \varphi_{2}=\left(\bar{\varphi}_{1} \otimes \bar{\varphi}_{2}\right) b(k, l)$ we obtain a $\left(\theta_{k}, \bar{\theta}_{k}\right)$-chain approximation of the map $F_{1} \times F_{2}$. This proves part (a) of Definition I.9.

Let $n \in \mathbb{N}$; there is an integer $k \geq n$ (again we assume $k$ to be independent of $i$ ) such that for integers $m_{1} \geq l_{1} \geq m \geq l \geq k$, and $\varphi_{i}=\bar{\varphi}_{i} b_{k}\left(l, l_{1}\right) \in A\left(F_{i}\right)_{l}, \psi_{i}=\bar{\psi}_{i} b_{i}\left(m, m_{1}\right) \in A\left(F_{i}\right)_{m}$ the chain maps $\bar{\varphi}_{i} \chi_{i}\left(m_{1}, l_{1}\right)$ and $\bar{\chi}_{i}(m, l) \bar{\psi}_{i}$ are chain homotopic with homotopies $D_{i}, i=1,2$, satisfying the condition (b) of Definition I.9 for the A-system $A\left(F_{i}\right)$, respectively. According to [8, p. 163] the chain maps

$$
\left(\bar{\varphi}_{1} \otimes \bar{\varphi}_{2}\right) \chi\left(m_{1}, l_{1}\right) \quad \text { and } \quad \bar{\chi}(m, l)\left(\bar{\psi}_{1} \otimes \bar{\psi}_{2}\right)
$$

are homotopic via the chain homotopy

$$
D=D_{1} \otimes\left(\bar{\varphi}_{2} \chi_{2}\left(m_{1}, l_{1}\right)\right)+\left(\chi_{1}(m, l) \bar{\psi}_{1}\right) \otimes D_{2} .
$$

This homotopy satisfies the condition (b) of Definition I.9. Thus $A\left(F_{1}\right) \otimes A\left(F_{2}\right)$ is an A-system for the map $F_{1} \times F_{2}$, with coefficients in the field $\mathbb{F}$, and with respect to the f.s.b.d. $\theta$ and $\bar{\theta}$. We call it the tensor product of the A-systems $A\left(F_{1}\right)$ and $A\left(F_{2}\right)$. Finally, let $\varphi_{1} \otimes \varphi_{2} \in A\left(F_{1}\right)_{k} \otimes A\left(F_{2}\right)_{k}$; then $K I \varphi_{1} \otimes \varphi_{2}=\left(K I \varphi_{1}\right)\left(K I \varphi_{2}\right)=$ $\left(K I A\left(F_{1}\right)\right)\left(K I A\left(F_{2}\right)\right)$.

Corollary 3. Let $F_{i}: K \rightarrow L_{i}$ be u.s.c. maps with A-systems $A\left(F_{i}\right)$ (coefficients in the field $\mathbb{F}$ ) with respect to f.s.b.d. $\hat{\tau}$ on $K$ and $\hat{\mu}^{i}$ on $L_{i}, i=1,2$. Then the map

$$
\Phi=\left(F_{1} \times F_{2}\right) \Delta: K \rightarrow L_{1} \times L_{2}
$$

has an A-system (with coefficients in $\mathbb{F}$ ) with respect to f.s.b.d. $\hat{\tau}$ and $\hat{\mu}^{1} \times \hat{\mu}^{2}$ with Kronecker index $\left(K I A\left(F_{1}\right)\right)\left(K I A\left(F_{2}\right)\right)$. Here $\Delta: K \rightarrow$ $K \times K$ denotes the diagonal map $\Delta(x)=(x, x)$.

Proof. Lemma 2 shows that the tensor product $A\left(F_{1}\right) \otimes A\left(F_{2}\right)$ is an A-system for the map $F_{1} \times F_{2}$ with respect to f.s.b.d. $\hat{\tau} \times \hat{\tau}, \hat{\mu}^{1} \times \hat{\mu}^{2}$.

Since the diagonal map $\Delta$ is single valued there exists an A-system $A(\Delta)$ with còefficients in $\mathbb{F}$ for $\Delta$ with respect to $\hat{\tau}$ and $\hat{\tau} \times \hat{\tau}$, and Kronecker index $K I A(\Delta)=1$, see [25, 26]. The composition $\left(A\left(F_{1}\right) \otimes A\left(F_{2}\right)\right) A(\Delta)$ of A-systems provides an A-system for the map $\Phi$ with Kronecker index $\left(K I A\left(F_{1}\right)\right)\left(K I A\left(F_{2}\right)\right)$, cf. [25, Lemma 2.16]. The chain maps in the A-system $\left(A\left(F_{1}\right) \otimes A\left(F_{2}\right)\right) A(\Delta)$ are compositions of chain maps of $A(\Delta)_{k}$ and $\left(A\left(F_{1}\right) \otimes A\left(F_{2}\right)\right)_{k}$, cf. [25, I.2] for the definition of the composition of A-systems. 
Let $K$ be a block complex with f.s.b.d. $\hat{\tau}=\left\{\tau_{k}\right\}$. Then the product f.s.b.d. $\hat{\tau} \times \hat{\tau}$ on $K \times K$ is $T$-equivariant (Remark I.6.4). Therefore $T$ induces a chain map

$$
T: C_{*}\left(\tau_{k}\right) \otimes C_{*}\left(\tau_{k}\right) \rightarrow C_{*}\left(\tau_{k}\right) \otimes C_{*}\left(\tau_{k}\right)
$$

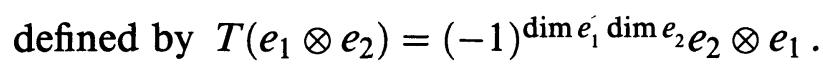

Corollary 4. Let $F: K \rightarrow L$ be an u.s.c. map and $A(F)$ an $A$ system (with coefficients in $\mathbb{F}$ ) for $F$ with respect to given f.s.b.d. $\hat{\tau}$, $\hat{\mu}$ on $K$ and $L$, respectively. Then there exists an A-system with coefficients in $\mathbb{F}$ for $F \times F$ w.r.t. $\hat{\tau} \times \hat{\tau}, \hat{\mu} \times \hat{\mu}$ which is T-equivariant, with Kronecker index $(K I A(F))(K I A(F))$.

Proof. Let $A_{T}(F \times F)_{k}=\left\{\varphi \otimes \varphi \mid \varphi \in A(F)_{k}\right\}$ and $A_{T}(F \times F)=$ $\left\{A_{T}(F \times F)_{k} \mid k \in \mathbb{N}\right\}$. Obviously $A_{T}(F \times F) \subset A(F) \otimes A(F)$. Since $A(F) \otimes A(F)$ is an A-system for $F \times F$ then $A_{T}(F \times F)$ is an A-system and it is $T$-equivariant; and $K I A_{T}(F \times F)=(K I A(F))(K I A(F))$.

3. Borsuk-Ulam theorem. In this subsection we prove the BorsukUlam theorem for u.s.c. maps having A-systems with coefficients in $\mathbb{Z}_{2}$.

Proposition 5. Let $F: S^{n} \rightarrow D_{a}^{n}$ be an u.s.c. map with A-system (coefficients in $\mathbb{Z}_{2}$ ), then the set

$$
B(F)=\left\{x \in S^{n} \mid F(x) \cap F(-x) \neq \varnothing\right\}
$$

is not empty.

Proof. Let $\hat{\tau}$ be a f.s.b.d. induced by an $\alpha$-equivariant block structure on $S^{n}$ and $\hat{\mu}$ be the f.s.b.d. induced by the cubic subdivision of $D_{a}^{n}$ (see Remark I.6.2). Without loss of generality we consider the A-system for $F$ with respect to the f.s.b.d. $\hat{\tau}$ and $\hat{\mu}$.

On the space $D_{a}^{n} \times D_{a}^{n}$ we consider the map

$$
\bar{d}: D_{a}^{n} \times D_{a}^{n} \rightarrow D_{2 a}^{n}, \quad \text { with } \bar{d}(x, y)=x-y,
$$

and on $S^{n}$ the map

$$
\bar{\Delta}: S^{n} \rightarrow S^{n} \times S^{n}, \quad \text { with } \bar{\Delta}(x)=(x,-x) .
$$

On the spaces $S^{n} \times S^{n}$ and $D_{a}^{n} \times D_{a}^{n}$ acts the involution $T$ defined by $T(x, y)=(y, x)$. The map $\bar{\Delta}$ is $(\alpha, T)$-equivariant, the map $\bar{d}$ is $(T, \alpha)$-equivariant. Since the map $\bar{\Delta}$ is single valued there exists an $(\alpha, T)$-equivariant A-system (coefficients in $\left.\mathbb{Z}_{2}\right) A(\bar{\Delta})$. with respect to 
f.s.b.d. $\hat{\tau}$ and $\hat{\tau} \times \hat{\tau}, K I A(\bar{\Delta})=1$, this follows form Lemma IV.3 or from appropriate simplicial approximations of $\bar{\Delta}$.

For the map $\bar{d}$ there exists a $(T, \alpha)$-equivariant A-system $A(\bar{d})$ (coefficients in $\mathbb{Z}_{2}$ ) with respect to f.s.b.d. $\hat{\mu} \times \hat{\mu}$ and $\hat{\mu}$ and $K I A(\bar{d})=$ 1 ; again, this follows from appropriate simplicial approximations of $\bar{d}$. Corollary 4 guarantees the existence of a $T$-equivariant A-system $\left(\mathbb{Z}_{2}\right.$ coefficients) $A_{T}(F \times F)$ with respect to f.s.b.d. $\hat{\tau} \times \hat{\tau}$ and $\hat{\mu} \times \hat{\mu}$ with Kronecker index $(K I A(F))(K I A(F))$.

Assume that the set $B(F)$ is empty and consider the map

$$
\Psi=\bar{d}(F \times F) \bar{\Delta}: S^{n} \rightarrow D_{2 a}^{n} .
$$

The assumption $B(F)=\varnothing$ leads to

$$
(F \times F) \bar{\Delta}\left(S^{n}\right) \cap\left\{(x, y) \in D_{a}^{n} \times D_{a}^{n} \times D_{a}^{n} \mid x=y\right\}=\varnothing
$$

and the compactness of $(F \times F) \bar{\Delta}\left(S^{n}\right)$ ensures the existence of $k_{0} \in \mathbb{N}$ such that the sets

$$
N=S t^{2}\left((F \times F) \bar{\Delta}\left(S^{n}\right), \mu_{k_{0}} \times \mu_{k_{0}}\right)
$$

and

$$
D=S t^{2}\left(\left\{(x, x) \in D_{a}^{n} \times D_{a}^{n}\right\}, \mu_{k_{0}} \times \mu_{k_{0}}\right)
$$

are disjoint. Since $D$ is a $T$-equivariant block complex with block structure $\mu_{k_{0}} \times \mu_{k_{0}}$ then $N_{0}=\overline{D_{a}^{n} \times D_{a}^{n} \backslash D}$ is a $T$-equivariant block complex with block structure $\mu_{k_{0}} \times \mu_{k_{0}}$ and $N \subset N_{0}$.

Due to the assumption $B(F)=\varnothing$ it follows that $0 \notin \bar{d}\left(N_{0}\right)$ and there exists $l_{0} \in \mathbb{N}$ such that $S t^{2}\left(0, \mu_{l_{0}}\right) \cap \bar{d}\left(N_{0}\right)=\varnothing$, here 0 denotes the point $(0, \ldots, 0) \in D_{2 a}^{n}$. Let $D_{0}=\overline{D_{2 a}^{n} \backslash S t^{2}\left(0, \mu_{l_{0}}\right)}$. Then $D_{0}$ is an $\alpha$-equivariant block complex with block structure $\mu_{l_{0}}$. The map $\rho: D_{0} \rightarrow S^{n-1}$ defined by $\rho(x)=x /\|x\|$ is $\alpha$-equivariant. Since it is single valued there exists an $\alpha$-equivariant A-system (coefficients in $\left.\mathbb{Z}_{2}\right) A(\rho)$ for $\rho$ with respect to $\hat{\mu}^{\prime}=\left\{\mu_{l} \mid l \geq l_{0}\right\}$ and $\hat{\tau}$.

It follows from [25, Lemma 2.16] that the composition

$$
A(\rho) A(\bar{d}) A_{T}(F \times F) A(\bar{\Delta})
$$

is an A-system for the map $\rho \Psi: S^{n} \rightarrow S^{n-1}$ with respect to $\hat{\tau}^{\prime}=$ $\left\{\tau_{i} \mid i \geq m_{0}\right\}, m_{0}=\max \left(l_{0}, k_{0}\right)$. This A-system with coefficients in $\mathbb{Z}_{2}$ is $\alpha$-equivariant. This contradicts Proposition 1.

4. Bourgin-Yang theorem. Let $F: S^{n+k} \rightarrow \mathbb{R}^{n}, k \geq 0$, be an u.s.c. map. The set $B(F)=\left\{x \in S^{n+k} \mid F(x) \cap F(-x) \neq \varnothing\right\}$ is compact and $\alpha$-equivariant. If $B(F) \neq \varnothing$ we consider the genus, $g(B(F), \alpha)$, of the space $(B(F), \alpha)$, where $\alpha$ is the antipodal map, see Remark II.2. 
Proposition 6. Let $F: S^{n+k} \rightarrow \mathbb{R}^{n}, k \geq 0$, be an u.s.c. map with an A-system (coefficients in $\mathbb{Z}_{2}$ ). Then $g(B(F), \alpha) \geq k$.

Proof. From Corollary 4 we have that $B(F) \neq \varnothing$. Following [12, Theorem 8.7], we assume $g(B(F), \alpha)=m<k$. Then there exists an $\alpha$-equivariant single valued map $f: B(F) \rightarrow S^{m}$. Let $\tilde{f}: S^{n+k} \rightarrow$ $\mathbb{R}^{m+1}$ be a continuous single valued extension of $f$, and consider the multivalued map

$$
\Psi=(F \times \tilde{f}) \Delta: S^{n+k} \rightarrow \mathbb{R}^{n} \times \mathbb{R}^{m+1}=\mathbb{R}^{m+n+1}
$$

defined by $\Psi(x)=F(x) \times \tilde{f}(x)$, where $\Delta(x)=(x, x)$ is the diagonal map. The map $\Psi$ is u.s.c. and Corollary 3 implies the existence of an A-system with coefficients in $\mathbb{Z}_{2}$ for $\Psi$. Since $m<k$ then $n+m+1 \leq$ $n+k$. From Proposition 5 one concludes the existence of a point $x_{0} \in S^{n+k}$ such that $\Psi\left(x_{0}\right) \cap \Psi\left(-x_{0}\right) \neq \varnothing$, which is equivalent to $F\left(x_{0}\right) \cap F\left(-x_{0}\right) \neq \varnothing$ and $\tilde{f}\left(x_{0}\right)=\tilde{f}\left(-x_{0}\right)$. But, then $x_{0} \in B(F)$ and $\tilde{f}\left(x_{0}\right)=f\left(x_{0}\right)=\tilde{f}\left(-x_{0}\right)=f\left(-x_{0}\right)$. Since $f: B(F) \rightarrow S^{m}$ is $\alpha$-equivariant we derive a contradiction.

COROLlary 7. Let $F: S^{n+k} \rightarrow \mathbb{R}^{n}, k \geq 0$, be an u.s.c. map with an $A$-system with coefficients in $\mathbb{Z}_{2}$. Then $\operatorname{dim} B(F) \geq k$.

Proof. This is a consequence of $\operatorname{dim} X \geq g(X, \gamma)$ [30], for any space with a free involution $\gamma$ and of Proposition 6.

\section{Existence of A-systems, fixed point index.}

1. A-systems. In this section we prove the existence of A-systems with coefficients in $\mathbb{F}$ for $\left(\gamma_{1}, \gamma_{2}\right)$-equivariant $m$-acyclic maps (w.r.t. a field $\mathbb{F}$ ), and $\gamma_{1}$ a free involution, see I.3 for the notation.

LeMma 1. Let $(K, \hat{\tau})$ and $(L, \hat{\mu})$ be compact polyhedra with a given f.s.b.d., furthermore, let $F: K \rightarrow L$ be a $\left(\gamma_{1}, \gamma_{2}\right)$-equivariant m-acyclic map (w.r.t. a field $\mathbb{F}), \gamma_{1}$ a free involution, and multiplicity $m(F)=a \in \mathbb{F}$. Let $n, k_{0}, l_{0} \in \mathbb{N}$. There are numbers $0<k_{0}<\cdots<$ $k_{n+1} ; \quad 0<l_{0}<\cdots<l_{n+1} \in \mathbb{N}$ such that for any block $e \in \tau_{k_{1}}$ there exists an integer $l_{i-1}(e)$ and a point $a(e) \in K$ such that

(a) $l_{i-1}<l_{i-1}(e)<l_{i}$ for $i=1, \ldots, n+1$.

(b) The covering $\left\{S t^{2}\left(y, l_{i}(e)\right) \mid y \in L\right\}$ is finer than the covering $\left\{S t\left(y, l_{i}\right) \mid y \in L\right\}$ and $e \subset S t\left(a(e), k_{i-1}\right)$ (cf. Remark I.7.2).

(c) $\gamma_{1}(a(e))=a\left(\gamma_{1}(e)\right)$ and $l_{i-1}(e)=l_{i-1}\left(\gamma_{1}(e)\right)$. 
(d) $F\left(S t^{2}\left(e, k_{i}\right)\right) \subset S t\left(F(a(e)), l_{i-1}(e)\right)$, and if $F(a(e))=C_{1}(a(e))$ $\cup \cdots \cup C_{s}(a(e)), s=s(a(e))$, then for all $u \neq v, u, v=1, \ldots, s$,

$$
S t^{2}\left(C_{u}(a(e)), l_{i-1}(e)\right) \cap S t^{2}\left(C_{v}(a(e)), l_{i-1}(e)\right)=\varnothing,
$$

and

$$
m\left(a(e), C_{u}(a(e))\right)=\sum_{C(x) \subset S t\left(C_{u}(a(e)), l_{i-1}(e)\right)} m(x, C(x)) .
$$

(e) The inclusion $S t^{2}\left(C_{u}(a(e)), l_{i-1}(e)\right) \subset S t\left(C_{u}(a(e)), l_{i-1}\right)$ induces the zero homomorphism in the reduced homology with coefficients in $\mathbb{F}$.

The proof is immediate from [25, Lemma B], [26, Lemma 2] and [9, Lemma 4.1].

LEMMA 2. Let the assumptions of Lemma 1 be satisfied. For any $\tau_{k} \in \hat{\tau}, \mu_{l} \in \hat{\mu}$ there exists a $\bar{k}>k$, and a chain map

$$
\varphi: C_{*}(K, \bar{k}) \rightarrow C_{*}(L, l)
$$

such that

(a) $\varphi$ is $\left(\gamma_{1}, \gamma_{2}\right)$-equivariant,

(b) $K I \varphi=m(F)$,

(c) for any block $e \in \tau_{\bar{k}}$ there exists a point $t(e) \in K$ such that

$$
\begin{gathered}
e \in S t(t(e), k), \\
|\varphi(e)| \subset S t(F(t(e)), l), \\
t\left(\gamma_{1}(e)\right)=\gamma_{1}(t(e)) .
\end{gathered}
$$

Proof. Applying Lemma 1 for $k_{0}=k, l_{0}=l, n=\operatorname{dim} K$ we set $\bar{k}=k_{n+1}$. We shall construct successively chain maps

$$
\varphi^{j}:\left(C_{*}((K, \bar{k}))^{(j)}\right) \rightarrow C_{*}\left(L, l_{n-j}\right),
$$

where $(K, \bar{k})^{(j)}$ denotes the $j$-skeleton of $(K, \bar{k})$, such that $\varphi^{j}$ is $\left(\gamma_{1}, \gamma_{2}\right)$-equivariant, and for any block $e \in \tau_{\bar{k}}$ with $\operatorname{dim} e \leq j$ there exists a point $t(e) \in K$ such that $e \in S t\left(t(e), k_{n-j}\right), t\left(\gamma_{1}(e)\right)=$ $\gamma_{1}(t(e))$. If

$$
F(t(e))=C_{1}(t(e)) \cup \cdots \cup C_{s}(t(e)), \quad s=s(t(e)),
$$

then $\varphi^{j}(e)=\varphi^{j}(e)_{1}+\cdots+\varphi^{j}(e)_{s}$ with $\left|\varphi^{j}(e)_{u}\right| \subset S t\left(C_{u}(t(e)), l_{n-j}\right)$ and $K I \varphi^{j}(e)_{u}=m\left(t(e), C_{u}(t(e))\right)$ for all $u=1, \ldots, s$. 
Step 1 . We start with $j=0$. Let $e \in \tau_{\bar{k}}$ and $\operatorname{dim} e=0$; Lemma 1 for $i=n+1$ guarantees the existence of a point $a(e) \in K$ such that (a)-(e) of Lemma 1 hold. We set $t(e)=a(e)$ and $t\left(\gamma_{1}(e)\right)=\gamma_{1}(t(e))$. Let $a_{n}$ be a zero-dimensional block in $S t\left(C_{u}(t(e)), l_{n}(e)\right)$, where $C_{u}(t(e)), u=1, \ldots, s=s(t(e))$, denotes a connected component of $F(t(e))$. Therefore

$$
\varphi^{0}(e)=\sum_{u=1}^{s} \varphi^{0}(e)_{u}
$$

with

$$
\varphi^{0}(e)_{u}=\chi\left(l_{n}(e), l_{n}\right) m\left(t(e), C_{u}(t(e))\right) a_{u}
$$

is well defined and satisfies the above conditions.

Step 2. Let $j=1$ and $e \in \tau_{\bar{k}}$ with $\operatorname{dim} e=1$ and $\bar{e}=\left|e, k_{n}\right|$ (support of the block $e$ in $\tau_{k_{n}}$ ). Due to Lemma 1 there exits an $a(\bar{e}) \in K$ and $l_{n-1}(\bar{e})$ such that (a)-(e) of Lemma 1 hold; define $t(e)=a(\bar{e})$ and $t\left(\gamma_{1}(e)\right)=\gamma_{1}(t(e))$.

Let $\partial e=e_{1}-e_{0}$ and $\varphi^{0}\left(e_{\nu}\right)=\sum_{u=1}^{s} \varphi^{0}\left(e_{\nu}\right)_{u}, s=s\left(t\left(e_{\nu}\right)\right)$, for $\nu=0,1 . \varphi^{0}\left(e_{\nu}\right)_{u}$ is a chain in $S t\left(C_{u}\left(t\left(e_{\nu}\right)\right), l_{n}\right)$. Furthermore,

$$
S t\left(F\left(t\left(e_{\nu}\right)\right), l_{n}\right) \subset S t^{2}\left(F(a(\bar{e})), l_{n-1}(\bar{e})\right)
$$

and

$$
\left|\varphi^{0}\left(e_{\nu}\right)\right| \subset S t^{2}\left(F(a(\bar{e})), l_{n-1}(\bar{e})\right)
$$

Now

$$
S t^{2}\left(F(a(\bar{e})), l_{n-1}(\bar{e})\right)=\bigcup_{r=1}^{s^{*}} S t^{2}\left(C_{r}(a(\bar{e})), l_{n-1}(\bar{e})\right), \quad s^{*}=s(a(\bar{e})),
$$

and we consider $\varphi^{0}\left(e_{\nu}\right)=\sum_{r=1}^{s^{*}} \varphi^{0}\left(e_{\nu}\right)^{r}$ with

$$
\varphi^{0}\left(e_{\nu}\right)^{r}=\sum_{u \in I_{r}} \varphi^{0}\left(e_{\nu}\right)_{u}
$$

where $I_{r}=\left\{u \mid C_{u}\left(t\left(e_{\nu}\right)\right) \subset S t^{2}\left(C_{r}(a(\bar{e})), l_{n-1}(\bar{e})\right)\right\}$, which gives

$$
K I \varphi^{0}\left(e_{\nu}\right)^{r}=\sum_{u \in I_{r}} m\left(t\left(e_{\nu}, C_{u}\left(t\left(e_{\nu}\right)\right)\right)\right)=m\left(a(\bar{e}), C_{r}(a(\bar{e}))\right) .
$$

Then $\chi\left(l_{n}, l_{n-1}\right)\left(\varphi^{0}\left(e_{0}\right)^{r}-\varphi^{0}\left(e_{1}\right)^{r}\right)$ is a chain in the block complex $S t\left(F(a(\bar{e})), l_{n-1}\right)$ with Kronecker index zero. Therefore there exists a chain $c_{r}=\varphi^{1}(e)_{r} \in S t\left(F(a(\bar{e})), l_{n-1}\right)$ such that

$$
\partial c_{r}=\chi\left(l_{n}, l_{n-1}\right)\left(\varphi^{0}\left(e_{0}\right)^{r}-\varphi^{0}\left(e_{1}\right)^{r}\right),
$$


and define $\varphi^{1}(e)=\sum_{r=1}^{s^{*}} \varphi^{1}(e)_{r}$ and $\varphi^{1}\left(\gamma_{1}(e)\right)=\gamma_{2}\left(\varphi^{1}(e)\right)$. For a zero-dimensional block $e$ we define $\varphi^{1}(e)=\chi\left(l_{n}, l_{n-1}\right) \varphi^{0}(e)$. The homomorphism $\varphi^{1}$ is a chain map which satisfies the above conditions.

Now it is clear how to proceed for $j>1$, see [25, Lemma 3.5], [26, Lemma 3], [9, Theorem 4.3].

REMARK 1 . If $F$ is an $m$-point map (or $m$-acyclic map, such that all connected components of $F(x)$ are $R_{\delta}$-sets, cf. [13]), then the above construction holds even for rings $\mathbb{A}$, i.e. in Lemmata 1 and 2 the field $\mathbb{F}$ may be replaced by the ring $\mathbb{A}$.

LEMMA 3. Let the map $F$ be as in Lemma 1, with f.s.b.d. $\hat{\tau}$ and $\hat{\mu}$. Then there exists $a\left(\gamma_{1}, \gamma_{2}\right)$-equivariant A-system with coefficients in $\mathbb{F}$ for $F$ such that $K I A(F)=m(F)$.

The proof follows directly from the above lemmata and the proofs in [25, Lemma 3.8], [9, Proposition 5.3].

REMARK 2. 1 . For $m$-point maps $F$, Lemma 3 implies the existence of A-systems with coefficients in a ring $\mathbb{A}$.

2. Let $\Phi: K \rightarrow L$ such that there exists an $m$-acyclic map (w.r.t. $\mathbb{F}) \quad F: K \rightarrow L$ with $F(x) \subset \Phi(x)$, for all $x \in K$, i.e., $F$ is a selector of the map $\Phi$. Then $\Phi$ possesses an A-system with coefficients in $\mathbb{F}$, and Lemma 3 holds for $\Phi$.

3. Let $F: K \rightarrow L$ be an $m$-acyclic map (w.r.t. $\mathbb{Z}$ ) and multiplicity function $m \geq 0$ and multiplicity $n>0$. Then $F$ may be decomposed into an u.s.c. acyclic map $\bar{F}: K \rightarrow S P^{n} L$ (w.r.t. $\mathbb{Z}$ ) and an $m$-point map $\pi: S P^{n} L \rightarrow L$ with multiplicity $n$, where (cf. Definition I.2)

$$
\bar{F}(x)=\left\{x_{1}^{k_{1}} \cdots x_{s}^{k_{s}} \mid x_{i} \in C_{i}(x), k_{i}=m\left(x, C_{i}(x)\right)\right\}, \quad s=s(x),
$$

and

$$
\pi\left(x_{1}^{l_{1}} \cdots x_{s}^{l_{s}}\right)=\left\{x_{1}, \ldots, x_{s}\right\} .
$$

Then $F=\pi \bar{F}$ and $F$ has an A-system with coefficients in $\mathbb{F}$, because $\bar{F}$ has one [25] and $\pi$ has one induced by a simplicial approximation of $\pi$ in [20].

4. For the maps in [5], [7], [16], the A-system can be constructed using simplicial approximation developed in [5], [7], [16], resp.

5. Let $p: K \rightarrow L$ be a continuous single valued map, $K, L$ finite polyhedra. We say that $p$ is a map with multiplicity (w.r.t. $\mathbb{A}$ ) if the map $p^{-1}: L \rightarrow K$ is an $m$-acyclic map (w.r.t. $\mathbb{A}$ ). If $p^{-1}$ is 
an $m$-point map it is sufficient to work with coefficients in a ring $\mathbb{A}$, otherwise the coefficients should be in a field $\mathbb{F}$. Let $A\left(p^{-1}\right)$ be the A-system for $p^{-1}$ constructed in Lemma 3. Every chain map $\varphi \in$ $A\left(p^{-1}\right)$ induces a homomorphism $\varphi_{*}: H_{*}(L, \mathbb{A}) \rightarrow H_{*}(K, \mathbb{A})$ which is a transfer homomorphism for the map $p$, i.e. $p_{*} \varphi_{*}=m\left(p^{-1}\right) i d$, cf. [28]. Therefore, the homomorphism $p_{*}: H_{*}(K, \overline{\mathbb{A}}) \rightarrow H_{*}(L, \overline{\mathbb{A}})$ is an epimorphism ( $\overline{\mathbb{A}}$ is the quotient field of the ring $\mathbb{A}$.) We will not go into more details.

Proposition 4. Let $F:\left(S^{n}, \alpha\right) \rightarrow\left(S^{m} \alpha\right)$ be an $\alpha$-equivariant $m$ acyclic map w.r.t. $\mathbb{Z}_{2}$; then $m \geq n$.

Proof. Lemma 3 implies the existence of an A-system for $F$ with coefficients in $\mathbb{Z}_{2}$ and Proposition III.1 yields $m \geq n$.

Proposition 5. Let $F: S^{n+k} \rightarrow \mathbb{R}^{n}, k \geq 0$, be an m-acyclic map w.r.t. $\mathbb{Z}_{2}$. then $\operatorname{dim}\left\{x \in S^{n+k} \mid F(x) \cap F(-x) \neq \varnothing\right\} \geq k$.

Proof. The existence of an A-system for $F$ with $\mathbb{Z}_{2}$-coefficients and Corollary III. 7 prove the assertion.

2. Fixed point index. For every map $\Phi: K \rightarrow K$ which has an Asystem $A(\Phi)$ with coefficients in the field $\mathbb{F}$ there exists a fixed point index defined in [25, Definition II.2.6]. Let $U$ be an open subset in $K$ such that $\bar{U}$ is a subcomplex in some $\tau_{k} \in \hat{\tau}$. Suppose $x \notin \Phi(x)$ for all $x \in \partial U$, i.e., the triple $(K, \Phi, U)$ is admissible.

Definition 6. The fixed point index $K_{A}(K, \Phi, U)$ is defined as

$$
I_{A}(K, \Phi, U)=\Lambda\left(\pi\left(\left.\varphi\right|_{\bar{U}}\right)\right)
$$

where $\varphi \in A(\Phi)_{l}, \pi: C_{*}\left(K, \tau_{l}\right) \rightarrow C_{*}\left(\bar{U}, \tau_{l}\right)$ is a homomorphism (projections) such that $\pi(e)=0$ for $e \notin \bar{U}$ and $\pi(e)=e$ for $e \in \bar{U}$, $e$ is a block in $\tau_{l}(l \geq k)$, and $\Lambda\left(\pi\left(\left.\varphi\right|_{\bar{U}}\right)\right)$ is the Lefschetz number of the homomorphism $\pi\left(\left.\varphi\right|_{\bar{U}}\right)$.

This definition does not depend on $l, \pi$ and $\tau$ for sufficiently large $l \in \mathbb{N}$ [25, Lemmata I.1.5 and II.2.5].

The fixed point index $I_{A}(K, \Phi, U)$ has all properties, i.e., additivity, homotopy invariance, normalization, commutativity, mod $p$ property, see [25]. Let $\mathscr{M}(\mathbb{F})$ be the set of $m$-acyclic maps of polyhedra w.r.t. the field $\mathbb{F}$. Let $F \in \mathscr{M}(\mathbb{F}), F: K \rightarrow K$, and $U$ an open subset in $k$ such that the triple $(K, F, U)$ is admissible. Applying 
Lemma 3 to the map $F, \gamma_{1}$ and $\gamma_{2}$ being the identity, we obtain an A-system $A(F)$ with $K I A(F)=m(F)=1$.

Definition 7. The fixed point index $i(K, F, U)$ is defined as

$$
i(K, F, U)=I_{A}(K, F, U)
$$

w.r.t. A-system $A(F)$ for every $F \in \mathscr{M}(\mathbb{F})$.

For $m$-acyclic maps the index $i(K, F, U)$ is independent from the choice of A-system $A(F)$, since $K I A(F)=1$, see [25, 26].

Proposition 8. Let $F \in \mathscr{M}(\mathbb{F})$, i.e., $F$ is an m-acyclic map w.r.t. the field $\mathbb{F}$. Then the fixed point index $i(K, F, U)$ has the following properties:

(a) Homotopy invariance. Let $H \in \mathscr{M}(\mathbb{F}), H: \bar{U} \times I \rightarrow K$, be a homotopy joining the maps $\left.\Phi_{1}\right|_{\bar{U}},\left.\Phi_{2}\right|_{\bar{U}} \in \mathscr{M}(\mathbb{F})$ such that for all $t \in I$ and $H_{t}: U \rightarrow K, H_{t}(x)=H(x, t)$, the triple $\left(K, H_{t}, U\right)$ is admissible. Then

$$
i\left(K, \Phi_{1}, U\right)=i\left(K, \Phi_{2}, U\right) .
$$

(b) Additivity. Let $\Phi \in \mathscr{M}(\mathbb{F}), \Phi: K \rightarrow K$, and $U_{1}, U_{2}$ be open disjoint subsets of $U$. If $\operatorname{Fix}\left(\left.\Phi\right|_{U}\right) \subset U_{1} \cup U_{2}$ then

$$
i(K, \Phi, U)=i\left(K, \Phi, U_{1}\right)+i\left(K, \Phi, U_{2}\right) \text {. }
$$

(c) Normalization.

$$
i(K, \varphi, K)=\Lambda(A(\Phi)) ;
$$

here $\Lambda(A(\Phi))$ denotes the Lefschetz number $\Lambda(\varphi)$ of a chain map $\varphi \in A(\Phi)_{k}$.

(d) Commutativity. Let $\Phi_{1}: K \rightarrow L, \Phi_{2}: L \rightarrow K$ be in $\mathscr{M}(\mathbb{F})$, and let $\left(K, \Phi_{2} \Phi_{1}, U\right)$ and $\left(L, \Phi_{1} \Phi_{2}, \Phi_{2}^{-1}(U)\right)$ be admissible. Assume that for all $y \in \operatorname{Fix}\left(\Phi_{1} \Phi_{2}\right) \backslash \Phi_{2}^{-1}(U)$ :

$$
\Phi_{2}(y) \cap \operatorname{Fix}\left(\left.\Phi_{2} \Phi_{1}\right|_{\bar{U}}\right)=\varnothing .
$$

Then

$$
I_{A}\left(K, \Phi_{2} \Phi_{1}, U\right)=I_{A}\left(L, \Phi_{1} \Phi_{2}, \Phi_{2}^{-1}(U)\right),
$$

where the fixed point index is defined with the A-systems $A\left(\Phi_{2}\right) A\left(\Phi_{1}\right)$, $A\left(\Phi_{1}\right) A\left(\Phi_{2}\right)$, respectively.

(e) Mod p-property. Let $p \in \mathbb{N}$ be prime and let $\Phi \in \mathscr{M}\left(\mathbb{Z}_{p}\right)$, $(K, \Phi, U),\left(K, \Phi^{p}, U\right)$ be admissible. Assume that for all $y \in$ $\operatorname{Fix}\left(\Phi^{p}\right) \backslash U$ :

$$
\Phi^{k}(y) \cap \operatorname{Fix}\left(\Phi^{p}(\bar{U})\right)=\varnothing, \quad 1 \leq k<p
$$


Then

$$
i(K, \Phi, U)=I_{A}\left(K, \Phi^{p}, U\right) \text { in } \mathbb{Z}_{p}
$$

Here the fixed point index for $\Phi^{p}$ is defined with the A-system $A(\Phi) \cdots A(\Phi)$ (p-times).

(f) Multiplicity. Let $\Phi_{i}: K_{i} \rightarrow L_{i}, \Phi_{i} \in \mathscr{M}(\mathbb{F})$, and $\left(K_{i}, \Phi_{i}, U_{i}\right)$ be admissible. Then

$$
i\left(K_{1} \times K_{2}, \Phi_{1} \times \Phi_{2}, U_{1} \times U_{2}\right)=i\left(K_{1}, \Phi_{1}, U_{1}\right) i\left(K_{2}, \Phi_{2}, U_{2}\right) .
$$

Here the index for $\Phi_{1} \times \Phi_{2}$ is defined with the A-system $A\left(\Phi_{1}\right) \otimes A\left(\Phi_{2}\right)$, see III.2, and is independent from the choice of A-systems.

Proof. The properties (a)-(e) follow from [25]: Homotopy invariance from II.15, additivity from II.2.8, normalization from Definition 5, commutativity from II.2.17, mod- $p$-property from II.2.19.(f), and multiplicity from [26, III.9].

REMARK 3. 1. For maps $f: K \rightarrow S P^{n} K$ the fixed point index is defined in [18].

2. In [9] the fixed point index for $(1, n)$-valued maps $F$, i.e., $F(x)$ consists of 1 or $n$ acyclic components, is defined; for $n$-valued maps see [24].

3. For the composition of $m$-maps see [5], [16].

4. The $m$-point maps $F$ (w.r.t. $\mathbb{Z}$ ), or more generally $m$-acyclic maps for which the connected components of $F(x)$ are $R_{\delta}$-sets, [13], have A-systems w.r.t. $\mathbb{Z}$ (Remark 2.1). Therefore the fixed point index $i(K, F, U)$ is integer valued.

The fixed point index for this kind of map with nonnegative multiplicity is constructed in [13].

\section{REFERENCES}

[1] E. Begle, The Vietoris mapping theorem for bicompact spaces, Ann. of Math. (2), 51 (1950), 534-543.

[2] C. Berge, Espaces Topologiques Fonctions Multivoques, Dunod, Paris, 1959.

[3] Y. Borisovič, E. Gelman, A. Myškis, and V. Obuhovskii, Topological methods in fixed point theory for multivalued mappings, Russian Math. Surveys, 35 (1980), 65-143.

[4] R. Brown, The Lefschetz Fixed Point Theorem, Glenview, III, Scott Foresman and Company, 1971. 
[5] G. Conti and J. Pejsachowicz, Fixed point theorem for multi-valued weighted maps, Ann. Mat. Pura Appl. (4), 126 (1980), 319-341.

[6] S. Darbo, Grado topologico e theoremi di esistenza di punti uniti per transformazioni plurivalenti di besele, Rend. Sem. Mat. Univ. Padova, 19 (1950), 371-395.

[7] _ Theoria de l'omologia in una categoria die mappe plurivalenti ponderati, Rend. Sem. Math. Univ. Padova, 28 (1958), 188-220.

[8] A. Dold, Lectures on Algebraic Topology, Springer, Berlin, Heidelberg, New York, 1980.

[9] Z. Dzedzej, Fixed point index for a class of nonacyclic multivalued maps, Dissertationes Math., 253 (1985), 1-58.

[10] S. Eilenberg and D. Montgomery, Fixed point theorems for multivalued transformations, Amer. J. Math., 58 (1946), 214-222.

[11] G. Fournier and D. Violette, A fixed point index for compositions of multivalued maps in Banach spaces (to appear).

[12] K. Geba and L. Gorniewicz, On the Bourgin-Yang theorem for multivalued maps, I, Bull. Acad. Polon. Sci. Math., 34 (1986), 315-322.

[13] L. Gorniewicz, A. Granas, and W. Krysewski, Sur la methode de l'homotopie dans la théorie des points fixes pour les applications multivoques, C.R. Acad. Sci. Paris Sér. I, 307 (1988), 489-492; 308 (1989), 449-452.

[14] M. Henriksen and J. Isbell, On the continuity of the real roots of algebraic equations, Proc. Amer. Math. Soc., 4 (1953), 431-434.

[15] J. Jaworowski, Theorem on antipodes for multivalued mappings and fixed point theorems, Bull. Acad. Polon. Sci. Math., 4 (1956), 187-192.

[16] R. Jerrad, Homology with multiple valued functions, Trans. Amer. Math. Soc., 213 (1975), 407-427.

[17] M. Krasnoselskii, On the estimation of the number of critical points of functionals, Uspekhi Mat. Nauk, 7 (1952), 157-164. (Russian)

[18] S. Masih, On the fixed point index and the Nielsen fixed point theorem of symmetric product mappings, Fund. Math., 102 (1972), 143-158.

[19] C. Maunder, Algebraic Topology, London, 1970.

[20] C. Maxwell, Fixed points of symmetric product mappings, Proc. Amer. Math. Soc., 8 (1957), 808-815.

[21] J. Pejsachowicz, The homotopy theorem of weighted mappings, Bull. Un. Mat. Ital. B(5), 14 (1977), 702-721.

[22] B. O'Neill, Induced homology homomorphisms for set valued maps, Pacific J. Math., 7 (1957), 1179-1184.

[23] H. Schirmer, Fixed points, antipodal points and coincidence of $n$-acyclic valued multifunctions, Contemp. Math., 21 (1983), 207-212.

[24] __ An index and a Nielsen number for $n$-valued multifunctions, Fund. Math., 124 (1984), 207-219.

[25] H.-W. Siegberg and G. Skordev, Fixed point index and chain approximation, Pacific J. Math., 102 (1982), 455-486.

[26] G. Skordev, Multiplicity of the fixed point index for multivalued maps, Serdica Bulg. Math. J., 15 (1989), 160-170.

[27] E. Spanier, Algebraic Topology, McGraw Hill Company, New York, 1966.

[28] L. Smith, Transfer and ramified coverings, Math. Proc. Cambridge Philos. Soc., 93 (1983), 485-493.

[29] N. Steenrod and D. Epstein, Cohomology operations, Ann. of Math. Studies, no. 50, Princeton Univ. Press, 1962. 
[30] H. Steinlein, Borsuk's antipodal theorem and its generalizations: a survey, Meth. topol. en anal. non linéare, Comp. rend. coll. par A. Granas, Montreal, 1985, 166-235.

Received March 13, 1990. This paper was written while the second author was a research fellow sponsored by Alexander von Humbold Foundation.

UNIVERSITÄT BREMEN

D-2800 Bremen 33, Germany

AND

UNIVERSITY OF SOFIA

1126 SOFIA, BULgaria 


\section{PACIFIC JOURNAL OF MATHEMATICS EDITORS}

\author{
V. S. VARADARAJAN \\ (Managing Editor) \\ University of California \\ Los Angeles, CA 90024-1555-05 \\ Herbert Clemens \\ University of Utah \\ Salt Lake City, UT 84112 \\ F. Michael Christ \\ University of California \\ Los Angeles, CA 90024-1555 \\ THOMAS ENRIGHT \\ University of California, San Diego \\ La Jolla, CA 92093
}

Nicholas ERCOLANI

University of Arizona

Tucson, AZ 85721

R. FINN

Stanford University

Stanford, CA 94305

VAUGHAN F. R. JONES

University of California

Berkeley, CA 94720

Steven Kerckhoff

Stanford University

Stanford, CA 94305

\section{C. MOORE}

University of California

Berkeley, CA 94720

Martin ScharlemanN

University of California

Santa Barbara, CA 93106

HAROLD STARK

University of California, San Diego

La Jolla, CA 92093

\section{R. ARENS \\ ASSOCIATE EDITORS}

\begin{tabular}{|c|c|c|c|c|}
\hline R. ARENS & $\begin{array}{l}\text { E. F. BECKENBACH } \\
(1906-1982)\end{array}$ & B. H. NeumanN & $\begin{array}{c}\text { F. WolF } \\
(1904-1989)\end{array}$ & K. YoshidA \\
\hline \multicolumn{5}{|c|}{ SUPPORTING INSTITUTIONS } \\
\hline \multicolumn{2}{|c|}{ UNIVERSITY OF ARIZONA } & UNIVER: & OF OREG & \\
\hline \multicolumn{2}{|c|}{ UNIVERSITY OF BRITISH COLUMB } & & & \\
\hline \multicolumn{2}{|c|}{ CALIFORNIA INSTITUTE OF TECHNOLOGY } & OGY STANI & INIV & \\
\hline \multicolumn{2}{|c|}{ UNIVERSITY OF CALIFORNIA } & UNI & & \\
\hline \multicolumn{2}{|c|}{ MONTANA STATE UNIVERSITY } & UNIVE & OF T & \\
\hline \multirow{2}{*}{\multicolumn{2}{|c|}{ NIVERSITY OF NEVADA, RENO }} & UNIVE & OF U & \\
\hline & & WASH & N ST & ERSIT \\
\hline \multicolumn{2}{|c|}{$\begin{array}{l}\text { NEW MEXICO STATE UNIVERSITY } \\
\text { OREGON STATE UNIVERSITY }\end{array}$} & UNIVERS & OF WASHI & \\
\hline
\end{tabular}

The Supporting Institutions listed above contribute to the cost of publication of this Journal, but they are not owners or publishers and have no responsibility for its content or policies.

Mathematical papers intended for publication in the Pacific Journal of Mathematics should be in typed form or offset-reproduced (not dittoed), double spaced with large margins. Please do not use built up fractions in the text of the manuscript. However, you may use them in the displayed equations. Underline Greek letters in red, German in green, and script in blue. The first paragraph must be capable of being used separately as a synopsis of the entire paper. In particular it should contain no bibliographic references. Please propose a heading for the odd numbered pages of less than 35 characters. Manuscripts, in triplicate, may be sent to any one of the editors. Please classify according to the 1991 Mathematics Subject Classification scheme which can be found in the December index volumes of Mathematical Reviews. Supply name and address of author to whom proofs should be sent. All other communications should be addressed to the managing editor, or Elaine Barth, University of California, Los Angeles, California 90024-1555-05.

There are page-charges associated with articles appearing in the Pacific Journal of Mathematics. These charges are expected to be paid by the author's University, Government Agency or Company. If the author or authors do not have access to such Institutional support these charges are waived. Single authors will receive 50 free reprints; joint authors will receive a total of 100 free reprints. Additional copies may be obtained at cost in multiples of 50 .

The Pacific Journal of Mathematics (ISSN 0030-8730) is published monthly except for July and August. Regular subscription rate: $\$ 190.00$ a year (10 issues). Special rate: $\$ 95.00$ a year to individual members of supporting institutions.

Subscriptions, orders for numbers issued in the last three calendar years, and changes of address should be sent to Pacific Journal of Mathematics, P.O. Box 969, Carmel Valley, CA 93924, U.S.A. Old back numbers obtainable from Kraus Periodicals Co., Route 100, Millwood, NY 10546.

The Pacific Journal of Mathematics at P.O. Box 969, Carmel Valley, CA 93924 (ISSN 0030-8730) is published monthly except for July and August. Second-class postage paid at Carmel Valley, California 93924, and additional mailing offices. Postmaster: send address changes to Pacific Journal of Mathematics, P.O. Box 969, Carmel Valley, CA 93924.

PUBLISHED BY PACIFIC JOURNAL OF MATHEMATICS, A NON-PROFIT CORPORATION Copyright (C) 1992 by Pacific Journal of Mathematics 


\section{Pacific Journal of Mathematics}

Vol. 153, No. $2 \quad$ April, 1992

R. Ayala, Eladio Domínguez Murillo, Alberto Márquez Pérez and A.

Quintero, Lusternik-Schnirelmann invariants in proper homotopy

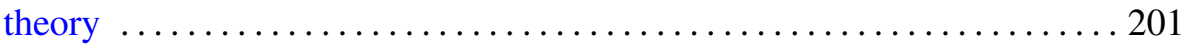

Hari Bercovici and Dan-Virgil Voiculescu, Lévy-Hinčin type theorems for multiplicative and additive free convolution $\ldots \ldots \ldots \ldots \ldots \ldots \ldots 217$

L. J. Bunce and Cho-Ho Chu, Compact operations, multipliers and Radon-Nikodým property in $J B^{*}$-triples $\ldots \ldots \ldots \ldots \ldots \ldots \ldots \ldots \ldots \ldots \ldots \ldots$

Marius Dadarlat, Gabriel Nagy, András Némethi and Cornel Pasnicu, Reduction of topological stable rank in inductive limits of $C^{*}$-algebras

François Dumas and Robert Vidal, Dérivations, et hautes dérivations, dans certains corps gauches de series de Laurent .................... 277

Mourad Ismail and Xin Li, On sieved orthogonal polynomials. IX:



X. T. Liang and Y. W. Lu, A Phragmén-Lindelöf theorem ...............299

Mark Stephen Reeder, On certain Iwahori invariants in the unramified principal series

Shohei Tanaka, On the representation of the determinant of Harish-Chandra's $C$-function of $\operatorname{SL}(n, \mathbb{R})$

Fritz von Haeseler and Guentcho Svetoslavov Skordev, Borsuk-Ulam theorem, fixed point index and chain approximations for maps with multiplicity 